
Cahiers
de ex Recherche
Fur les Droits
Fondamentaux

\section{Cahiers de la recherche sur les droits} fondamentaux

$7 \mid 2009$

L'universalisme des droits en question(s). La Déclaration universelle des Droits de l'homme, 60 ans après

\title{
L'article 5 de la Déclaration universelle des Droits de l'homme, substrat juridique du cadre international de lutte contre la torture
}

Isabelle Moulier

\section{OpenEdition}

Journals

Édition électronique

URL : https://journals.openedition.org/crdf/6687

DOI : $10.4000 /$ crdf.6687

ISSN : 2264-1246

Éditeur

Presses universitaires de Caen

Édition imprimée

Date de publication : 31 décembre 2009

Pagination : 87-106

ISBN : 978-2-84133-342-4

ISSN : $1634-8842$

Référence électronique

Isabelle Moulier, «L'article 5 de la Déclaration universelle des Droits de l'homme, substrat juridique du cadre international de lutte contre la torture », Cahiers de la recherche sur les droits fondamentaux [En ligne], 7 | 2009, mis en ligne le 15 décembre 2020, consulté le 14 novembre 2022. URL : http:// journals.openedition.org/crdf/6687 ; DOI : https://doi.org/10.4000/crdf.6687 


\title{
L'article 5 de la Déclaration universelle des Droits de l'homme, substrat juridique du cadre international de lutte contre la torture
}

\author{
Isabelle MOULIER \\ Maître de conférences en droit public à I'Université de Caen Basse-Normandie \\ Centre de recherche sur les droits fondamentaux et les évolutions du droit (CRDFED)
}

I. Le développement progressif des potentialités normatives de l'article 5

A. De l'interdiction à l'incrimination internationale de la torture

B. Une interdiction absolue en toutes circonstances

1. Une interdiction indérogeable

2. Une interdiction relevant du jus cogens

II. Le développement des mécanismes visant à prévenir la torture

III. De la répression à la réparation de la torture

A. L'universalisation de la répression de la torture

1. La consécration conventionnelle de la compétence universelle à l'égard de la torture

2. Le lien existant entre les normes fondamentales et la compétence universelle

3. La mise en œuvre concrète de la compétence universelle à l'égard de la torture

B. Le droit de la victime à la réparation du préjudice subi

Si la pratique de la torture fut dénoncée avec force par les écrivains et philosophes du XVIII ${ }^{\mathrm{e}}$ siècle, à l'instar de Voltaire $^{1}$ ou du criminaliste italien Cesare Beccaria ${ }^{2}$, sa prohibition par les mécanismes internationaux de protection des Droits de l'homme correspond cependant à une conquête relativement récente ${ }^{3}$. Rédigée dans l'immédiat

1. Voir Voltaire, Dictionnaire philosophique [1764], Paris, Gallimard (Folio), 2002, p. 502-503.

2. Voir C. Beccaria, Des délits et des peines [1764], Paris, Flammarion, 1991, § XVI, p. 95-104.

3. S'agissant du droit international humanitaire, applicable en cas de conflit armé, l'article 4 du Règlement annexé aux Conventions de La Haye de 1899 et 1907 concernant les lois et coutumes de la guerre sur terre, sans mentionner expressément la torture, dispose cependant que les prisonniers de guerre doivent être traités avec humanité, ce qui exclut clairement les traitements assimilables à la torture. Un article commun aux quatre Conventions de Genève pour la protection des victimes de la guerre du 12 août 1949, applicable en temps de conflit armé international, classe la « torture ou les traitements inhumains " parmi les infractions graves au droit international humanitaire, c'est-à-dire parmi les crimes de guerre (art. 50/51/130/147) L'article 75 du protocole I additionnel aux Conventions de Genève de 1977, relatif à la protection des victimes des conflits armés internationaux, précise que «la torture sous toutes ses formes, qu'elle soit physique ou mentale» est absolument prohibée. S'agissant des conflits armés non internationaux, l'article 3 commun aux quatre Conventions de Genève du 12 août 1949 inclut, dans la liste des règles minimales qui doivent être observées par toutes les 
de l'après-guerre, avec comme toile de fond le souvenir des «actes de barbarie qui révoltent la conscience de l'humanité» ${ }^{4}$, la Déclaration universelle des Droits de l'homme du 10 décembre 1948 constitue en effet le premier texte juridique international à énoncer, en termes généraux, l'interdiction de la torture. L'article 5 de la Déclaration dispose à cet égard que « [n]ul ne sera soumis à la torture, ni à des peines ou traitements cruels, inhumains ou dégradants ».

À l'origine, la Déclaration universelle des Droits de l'homme, issue d'une simple résolution de l'Assemblée générale des Nations unies, ne possédait pas de force juridique contraignante ${ }^{5}$ et se présentait comme «l'idéal à atteindre par tous les peuples et par toutes les Nations ${ }^{6}$. Soixante ans après l'adoption de la Déclaration universelle des Droits de l'homme, il n'est pas besoin de s'attarder longuement sur le fait que cet idéal est loin d'avoir été atteint : la pratique de la torture connaît en effet des formes renouvelées, notamment dans le cadre de la lutte contre le terrorisme, qu'il s'agisse des actes de torture ou traitements cruels, inhumains ou dégradants commis à l'encontre des prisonniers de Guantánamo ou au sein de la prison irakienne d'Abou Ghraïb ${ }^{7}$ en passant par les lieux de détention secrète qui constituent de véritables zones de non-droit ${ }^{8}$. Dans ce contexte, et face à l'existence d'un courant de pensée et d'une pratique préconisant un certain relativisme des valeurs face au paradigme sécuritaire, il paraît légitime de s'interroger sur la pertinence du cadre juridique de la lutte contre la torture envisagée en tant que violation flagrante des Droits de l'homme - élaboré depuis la première proclamation internationale de son interdiction dans la Déclaration.

Afin de pouvoir se prononcer sur le bien-fondé du cadre juridique international existant, il convient, dans un premier temps, d'examiner la signification juridique de cette interdiction qui s'est progressivement affermie pour déployer toutes ses potentialités normatives (I). Audelà de la proclamation théorique du droit, c'est cependant son application effective qui importe au premier chef.
Il conviendra ainsi de procéder, dans un second temps, à l'examen des mesures concrètes envisagées pour assurer le respect de l'interdiction de la torture qui présentent des aspects novateurs par rapport aux autres mécanismes existant en matière de protection des Droits de l'homme. Si, en amont, la prévention de la torture s'avère primordiale pour assurer le respect de l'interdiction (II), il importe également, en aval, que la répression des tortionnaires et la réparation du préjudice subi par les victimes soient pleinement assurées (III).

\section{Le développement progressif des potentialités normatives de l'article 5}

Initialement proclamée dans la Déclaration universelle des Droits de l'homme, l'interdiction de la torture a ensuite connu une croissance normative importante, l'article 5 de la Déclaration constituant le socle à partir duquel les contours juridiques de l'interdiction de la torture ont été élaborés et précisés par d'autres instruments internationaux. Sa criminalisation progressive (A) et l'énoncé de son caractère absolu (B) ont ainsi progressivement donné corps à l'interdiction.

\section{A. De l'interdiction à l'incrimination internationale de la torture}

L’interdiction de la torture proclamée dans la Déclaration a constitué un point départ et une référence incontournable pour l'élaboration d'un cadre juridique applicable à la lutte contre la torture qui s'est progressivement étoffé. L'article 5 de la Déclaration s'est en effet révélé porteur de grandes potentialités puisque l'interdiction de la torture a ensuite été reprise dans de nombreuses conventions régionales ou internationales, qu'il s'agisse de l'article 3 de la Convention européenne des Droits de l'homme de $1950^{9}$, de l'article 7 du Pacte international

parties au conflit, y compris dans un conflit armé non international, une interdiction concernant les «atteintes portées à la vie et à l’intégrité corporelle, notamment les mutilations, traitements cruels, tortures et supplices ». Le protocole II de 1977, additionnel aux Conventions de Genève et relatif à la protection des victimes des conflits armés non internationaux, prohibe enfin «les traitements cruels tels que la torture, les mutilations ou toutes autres formes de peines corporelles ». Enfin, aux termes des Statuts des Tribunaux pénaux internationaux pour l'ex-Yougoslavie et le Rwanda ainsi que celui de la Cour pénale internationale, les actes de torture peuvent constituer des crimes de guerre et/ou des crimes contre l'humanité.

4. Second considérant du préambule de la Déclaration universelle des Droits de l’homme. Doc. ONU, A/RES/217 A (III), 10 décembre 1948.

5. Lors de l'adoption de la Déclaration, René Cassin, délégué de la France à l'Assemblée générale, tint les propos suivants: «[1]a Déclaration a une grande portée morale. De plus, si elle n'est pas aussi puissante et astreignante qu'une convention, elle n'en a pas moins une valeur juridique car elle est formulée dans une résolution de l'Assemblée qui a une valeur juridique de recommandation : elle est le développement de la Charte qui a incorporé les Droits de l'homme dans le droit international positif. Dès lors, on ne peut pas dire que la Déclaration soit un instrument purement académique. Ce n'est qu'un instrument potentiel qui, cependant, n'enlève rien aux obligations déjà existantes en vertu de la Charte. » Propos cités par A. Kiss, Répertoire de la pratique française en matière de droit international public, Paris, Éditions du CNRS, 1966, t. II, $\$ 1117$. Sur le débat relatif à la valeur juridique de la Déclaration, voir E. Decaux, « De la promotion à la protection des Droits de l'homme. Droit déclaratoire et droit programmatoire », in La protection des Droits de l'homme et l'évolution du droit international, Colloque de Strasbourg, mai 1997, SFDI (éd.), Paris, Pedone, 1998, p. 80-119 (102-119).

6. Dernier considérant du préambule de la Déclaration.

7. M. Benvenisti, Abu Ghraib: the Politics of Torture, Berkeley, North Atlantic Books, 2004. De manière générale, voir J. Ross, «Sévices infligés à des prisonniers : la réponse légale apportée par les États-Unis à la question de la torture depuis le 11 septembre 2001 ", RICR, vol. 89, $\mathrm{n}^{\circ} 867$, septembre 2007, p. 561-590.

8. Voir en ce sens la résolution de l'Assemblée générale des Nations unies 60/148 du 16 décembre 2005 dans laquelle elle « rappelle à tous les États qu'une période prolongée de mise au secret ou de détention dans des lieux secrets peut faciliter la pratique de la torture et d'autres peines ou traitements cruels, inhumains ou dégradants et peut en soi constituer un tel traitement, et demande instamment à tous les États de respecter les garanties concernant la liberté, la sécurité et la dignité de la personne». Doc. ONU, A/RES/60/148, 16 décembre $2005, \S 11$.

9. «Nul ne peut être soumis à la torture, ni à des peines ou traitements inhumains ou dégradants. » 
relatif aux droits civils et politiques de $1966^{10}$, de l'article 5 de la Convention américaine des Droits de l'homme de $1969^{11}$ ou encore de l'article 5 de la Charte africaine des Droits de l'homme et des peuples de $1981^{12}$. L'élaboration de ce cadre conventionnel a ainsi permis que des obligations juridiques - consistant en des obligations juridiques d'abstention - se substituent aux obligations morales inscrites dans la Déclaration universelle.

L'interdiction formelle de la torture ne fut cependant pas à même d'empêcher certains États de pratiquer à plus ou moins grande échelle la torture, celle-ci se trouvant parfois érigée en véritable méthode de gouvernement. Or, au milieu des années 1970, il n'existait aucun texte spécifique relatif à la lutte contre la torture. De nombreuses organisations internationales non gouvernementales dénoncèrent ainsi, à partir de cette période, la persistance dans certains États de l'utilisation de la torture comme "l'un des rouages d'une machine contrôlée par les institutions politiques et destinée à éliminer toute dissidence ou opposition ${ }^{13}$. Elles n'eurent de cesse d'appeler l'attention de la communauté internationale sur la nécessité d'adopter des instruments spécifiques contre la torture et autres pratiques similaires ${ }^{14}$, en vue d'accroître l'efficacité du principe de leur interdiction. L'Assemblée générale des Nations unies devait partiellement combler cette lacune le 9 décembre 1975 en adoptant la Déclaration sur la protection de toutes les personnes contre la torture et autres peines ou traitements cruels, inhumains ou dégradants ${ }^{15}$. Bien que dépourvue de force juridique contraignante, cette résolution constitue le premier texte de portée universelle spécifiquement consacré à la torture. Il allait marquer une étape importante dans l'élaboration d'un futur instrument conventionnel, à vocation universelle.

Le 8 décembre 1977, « estimant qu'une nouvelle action internationale [était] nécessaire pour assurer à tous une protection appropriée contre la torture et autres peines ou traitements cruels, inhumains ou dégradants $»^{16}$, l'Assemblée générale pria en effet la Commission des Droits de l'homme de l'ONU d'élaborer un projet de convention contre la torture ${ }^{17}$, au regard des principes exposés dans sa Déclaration de 1975. Les travaux de la Commission débouchèrent sur l'adoption, par l'Assemblée générale des Nations unies, le 10 décembre 1984, de la Convention contre la torture et autres peines ou traitements cruels, inhumains ou dégradants ${ }^{18}$.

Si le principe de la prohibition de la torture n'a guère posé de difficultés en lui-même, il en va différemment s'agissant de sa définition. Il a en effet fallu attendre la résolution 3452 (XXX) de l'Assemblée générale du 9 décembre 1975 pour que la première définition juridique de la torture soit adoptée ${ }^{19}$. Cette définition fut ensuite reprise par un texte juridique contraignant, la Convention de 1984. Elle est considérée comme coutumière par les Tribunaux pénaux internationaux pour l'ex-Yougoslavie et pour le Rwanda ${ }^{20}$. Selon l'article premier de la Convention, la torture consiste en substance dans le fait d'infliger à une personne des souffrances aiguës, physiques ou morales, commises directement ou indirectement, par un agent de la fonction publique ou toute autre personne agissant à titre officiel, ou à son instigation ou avec son consentement exprès ou tacite, dans un but précis. Plusieurs éléments centraux doivent être retenus de cette définition ${ }^{21}$.

- La torture consiste en une souffrance aiguë qui peut être non seulement physique mais également mentale, ce qui inclut les méthodes modernes utilisées pour l'extorsion d'aveux ou de renseignements.

- La Convention ne vise que la torture «officielle», c'est-à-dire les faits de torture commis par des personnes agissant à titre officiel ou agissant avec le consentement de ces personnes. Elle n'a ainsi pas vocation à s'appliquer aux mêmes faits commis par des personnes privées.

10. Le Pacte étend la portée de l'interdiction aux expériences médicales ou scientifiques auxquelles une personne serait soumise sans son consentement.

11. Convention américaine relative aux Droits de l'homme (Pacte de San José de Costa Rica), San José, 22 novembre 1969, RTNU, vol. 1144, p. 123. Pour de plus amples développements, voir L. Burgorgue-Larsen, «La prohibition de la torture et ses équivalents dans le système interaméricain des Droits de l'homme", in La portée de l'article 3 de la Convention européenne des Droits de l'homme, C.-A. Chassin (éd.), Bruxelles, Bruylant, 2006, p. $23-46$.

12. Charte africaine des Droits de l'homme et des peuples, 27 juin 1981. Doc. OUA, CAB/LEG/67/3, rev. 5.

13. Z. Haquani, « La Convention des Nations unies contre la torture», RGDIP, n 1, 1986, p. 128.

14. Sur ce point, voir M. C. Bassiouni, «An Appraisal of Torture in International Law and Practice : The Need for an International Convention for the Prevention and Suppression of Torture ", RIDP, vol. 48, n 3-4, 1977, p. 17-114.

15. Doc. ONU, A/RES/3452 (XXX), 9 décembre 1975.

16. Doc. ONU, A/RES/32/62, 8 décembre $1977,3^{\mathrm{e}}$ considérant du préambule de la résolution.

17. Doc. ONU, A/RES/32/62, ibid., \$1.

18. Convention contre la torture et autres peines ou traitements cruels, inhumains ou dégradants, 10 décembre 1984, entrée en vigueur le 26 juin 1987 , RTNU, vol. 1465, p. 85. Au 17 décembre 2008, 146 États étaient parties à cette Convention. Sur cette Convention, voir J. H. Burgers et H. Danelius, The United Nations Convention against Torture. A Handbook on the Convention against Torture and Other Cruel, Inhuman or Degrading Treatment or Punishment, Dordrecht - Boston - Londres, Martinus Nijhoff Publishers - International Studies in Human Rights, 1988; C. Chanet, «La Convention des Nations unies contre la torture et autres peines ou traitements cruels, inhumains ou dégradants ", AFDI, 1984, p. 625-636; M. Novak, The United Nations Convention against Torture: a Commentary, Oxford, Oxford University Press, 2008 ; Z. Haquani, « La Convention des Nations unies contre la torture », RGDIP, $\mathrm{n}^{\circ} 1,1986$, p. 128-170.

19. Doc. ONU, A/RES/3452 (XXX), 9 décembre 1975.

20. Voir TPIR, Le Procureur c. Jean-Paul Akayesu, aff. n IT-96-4-T, Chambre de première instance I, 2 septembre 1998, \$593; TPIY, Le Procureur c. Zejnil Delalic, Zdravko Mucic alias «Pavo», Hazim Delic, Esad Landzo alias "Zenga», aff. n IT-96-21-T, Chambre de première instance II quater, jugement, 16 novembre 1998, \$ 459; TPIY, Le Procureur c. Anto Furundzija, aff. n IT-95-17/1-T10, Chambre de première instance II, jugement, 10 décembre 1998, $\$ 160$.

21. Pour un examen détaillé, voir R. ST. J. MacDonald, «International Prohibitions against Torture and Other Forms of Similar Treatment or Punishment", in International Law at a Time of Perplexity. Essays in Honour of Shabtai Rosenne, Y. Dinstein (éd.), Dordrecht, Kluwer Academic Publishers, 1989, p. 385-406. 
- La Convention exclut «la torture gratuite ${ }^{22}$ en ce sens que la torture doit avoir été pratiquée dans un certain but (obtention d'aveux, d'informations, intimidation, faire pression sur la personne elle-même ou sur un tiers...). Le texte conventionnel précise que la torture s'entend également d'actes commis «pour tout autre motif fondé sur une forme de discrimination quelle qu'elle soit ». Si cette dernière mention permet d'opérer une interprétation assez large du but poursuivi, la notion de discrimination, fondée sur une différence, demeure cependant juridiquement imprécise si elle n'est pas qualifiée (discrimination raciale, fondée sur le sexe...). Cette notion de discrimination est en outre susceptible de constituer une restriction dans la mesure où des faits de torture qui ne sont pas nécessairement fondés sur une discrimination, à l'instar de tortures pratiquées par des gardiens de prison sur des détenus, sont exclus du champ d'application de la Convention et notamment du champ de la répression qu'elle établit.

- Enfin, la torture ne s'étend pas à la douleur ou aux souffrances résultant de "sanctions légitimes ${ }^{23}$. Si ces termes ne sont pas définis plus avant par la Convention, il est toutefois possible de considérer, conformément à l'article premier, paragraphe 1, de la Déclaration de l'Assemblée générale de 1975, qu'ils s'entendent des «sanctions compatibles avec l'ensemble des règles minima pour le traitement des détenus».

Outre sa vocation universaliste, cette Convention est importante car elle est la seule parmi les instruments susmentionnés qui se bornaient à comporter des normes prohibitives, à consacrer la torture en tant qu'infraction internationale à part entière, soit, autrement dit, à criminaliser la torture, en établissant une sanction en cas de violation de la règle comportementale posée. Avant cette Convention, la torture ne constituait pas en effet une infraction pénale en droit international. Or, comme l'explique Michel Cosnard, «interdire un certain comportement est une chose, le criminaliser en est une autre. La première proposition correspond à l'essence même de la règle de droit, qui consiste à édicter un certain comportement, négatif en l'occurrence. La seconde tient à la sanction attachée à la violation de la règle comportementale. » ${ }^{24}$
La torture constitue désormais une infraction pénale autonome. L'article 4 de la Convention onusienne fait en effet obligation à tout État partie de veiller «à ce que tous les actes de torture constituent des infractions au regard de son droit pénal ${ }^{25}$ et de rendre « ces infractions passibles de peines appropriées qui prennent en considération leur gravité ${ }^{26}$. La complicité, la tentative et la participation doivent également être pénalement incriminées en tant qu'actes de torture ${ }^{27}$. C'est ainsi sous l'influence du droit international, à la suite de la ratification de la Convention onusienne de la torture en 1987, que la France a érigé ce comportement, à l'occasion de la réforme du Code pénal, en incrimination spécifique ${ }^{28}$. Avant cette date, il n'existait pas en France d'incrimination autonome de la torture qui ne constituait qu'une circonstance aggravante d'un certain nombre de crimes, en particulier du crime de séquestration. Si la torture n'est pas définie plus avant par le droit interne, le droit français opère cependant sur ce point un renvoi explicite à l'article premier de la Convention ${ }^{29}$. La Convention interaméricaine pour la prévention et la répression de la torture signée le 9 décembre 1985 à Cartagena de Indias (Colombie), entrée en vigueur le 28 février 1987, s'inscrit également dans le sens d'une criminalisation de la torture ${ }^{30}$.

\section{B. Une interdiction absolue en toutes circonstances}

L'interdiction de la torture consacre, selon la Cour européenne des Droits de l'homme, "l'une des valeurs fondamentales des sociétés démocratiques ${ }^{31}$. La prohibition de la torture s'avère en effet particulièrement importante: elle constitue non seulement une interdiction indérogeable (1) mais également une norme relevant du droit impératif (jus cogens) (2).

\section{Une interdiction indérogeable}

Au plan juridique, la singularité de la prohibition de la torture se traduit par l'énoncé d'une interdiction absolue, celle-ci n'étant susceptible d'aucune restriction ou dérogation. L'article 7 du Pacte international relatif aux droits civils et politiques, comme l'article 3 de la

22. C. Chanet, «La Convention des Nations unies contre la torture... », p. 627.

23. Article 1, paragraphe 1, de la Convention.

24. M. Cosnard, «La compétence universelle en matière pénale », in The Fundamental Rules of the International Legal Order: Jus Cogens and Obligations Erga Omnes, C. Tomuschat et J.-M. Thouvenin (éd.), Leyde, Nijhoff, 2006, p. 355-372 (355).

25. Article 4, paragraphe 1 , de la Convention.

26. Article 4, paragraphe 2, de la Convention.

27. Ibid.

28. Voir les articles 222-1 à 222-6 du nouveau Code pénal de 1992, entré en vigueur en 1994. L'article 222-1 dispose notamment que « [1] e fait de soumettre une personne à des tortures ou à des actes de barbarie est puni de quinze ans de réclusion criminelle». La peine passe à vingt ans en présence d'une circonstance aggravante.

29. Voir l'article 689-2 du Code de procédure pénale qui énonce que « [p] our l'application de la Convention contre la torture et autres peines ou traitements cruels, inhumains ou dégradants, adoptée à New York, le 10 décembre 1984, peut être poursuivie et jugée [...] toute personne coupable de tortures au sens de l'article $1^{\text {er }}$ de la Convention ".

30. Convention interaméricaine pour la prévention et la répression de la torture, signée le 9 décembre 1985, à Cartagena de Indias (Colombie), Série des traités de l'OEA, $\mathrm{n}^{\circ}$ 67. L'article 6 de la Convention énonce que " [1] es États parties s'assurent que tout acte ou toute tentative de torture constituent des crimes selon leur droit pénal ; ils établissent pour les punir des sanctions sévères tenant compte de leur gravité».

31. CEDH, Soering c. Royaume-Uni, req. $n^{\circ} 14038 / 88$, arrêt, 7 juillet 1989, $\$ 88$. 
Convention européenne des Droits de l'homme, font partie de ce que l'on dénomme le «noyau dur» des Droits de l'homme. L'interdiction de la torture relève en effet de la catégorie des «droits absolument intangibles » ${ }^{32}$ qui ne sont susceptibles d'aucune dérogation et qui s'appliquent en tous temps et en tous lieux, y compris « en cas de guerre ou en cas d'autre danger public menaçant la vie de la nation ${ }^{33}$. La non-dérogeabilité de l'interdiction de la torture est rappelée avec force par la Convention des Nations unies de 1984 contre la torture. Elle dispose en effet qu' : "[a]ucune circonstance exceptionnelle, quelle qu'elle soit, qu'il s'agisse de l'état de guerre ou de menace de guerre, d'instabilité politique intérieure ou de tout autre état d'exception, ne peut être invoquée pour justifier la torture ${ }^{34}$.»

Au regard de l'actualité récente, il n'est pas inutile de rappeler que cette interdiction absolue s'applique même en présence de circonstances exceptionnelles, à l'instar des actions menées dans le cadre de la lutte contre le terrorisme, comme en témoigne une jurisprudence constante de la Cour européenne des Droits de l'homme ${ }^{35}$. Si la Cour se déclare "parfaitement consciente des énormes difficultés que rencontrent à notre époque les États pour protéger leur population de la violence terroriste ${ }^{36}$, elle n'en considère pas moins que: " $[\ldots]$ même en tenant compte de ces facteurs, la Convention prohibe en termes absolus la torture ou les peines ou traitements cruels, inhumains ou dégradants, quels que soient les agissements de la victime. L'article 3 ne prévoit pas de restrictions, en quoi il contraste avec la majorité des clauses normatives de la Convention et des protocoles $n^{\circ} 1$ et 4 et il ne souffre aucune dérogation d'après l'article 15 même en cas de danger public menaçant la vie de la nation ${ }^{37}$.

Les lignes directrices du Comité des ministres du Conseil de l'Europe relatives aux Droits de l'homme et à la lutte contre le terrorisme sont d'ailleurs venues codifier la jurisprudence de la Cour européenne des Droits de l'homme sur ce point ${ }^{38}$.

Au niveau international, face aux tentatives de plus en plus nombreuses de légitimation du recours à la torture au nom d'un intérêt supérieur de protection de la sécurité nationale, l'Assemblée générale des Nations unies est également venue rappeler que l'interdit de la torture et pratiques équivalentes était absolu. Dans sa résolution 60/148 adoptée le 21 février 2006, l'Assemblée générale « rappelle que le droit d'être à l'abri de la torture et autres peines ou traitements cruels, inhumains ou dégradants est un droit intangible qui doit être protégé en toutes circonstances, y compris en période de conflit armé ou de troubles internationaux ou internes ${ }^{39}$.

D'autres instances ont par ailleurs rappelé que le droit international des Droits de l'homme avait également vocation à s'appliquer en présence de circonstances exceptionnelles, alors que les États-Unis ou encore Israël tentaient de dissocier le droit international des Droits de l'homme qui s'appliquerait en période dite "normale " et le droit international humanitaire qui s'appliquerait seul aux situations de conflit armé. Dans son avis consultatif rendu le 9 juillet 2004 dans l'affaire relative aux Conséquences juridiques de l'édification d'un mur dans le territoire

32. Pour reprendre l'expression d'O. de Frouville, L'intangibilité des Droits de l'homme en droit international: régime conventionnel des Droits de l'homme et droit des traités, Paris, Pedone, 2004, p. 117.

33. Voir l'article 15 de la Convention européenne des Droits de l'homme : «1. En cas de guerre ou en cas d'autre danger public menaçant la vie de la nation, toute Haute Partie contractante peut prendre des mesures dérogeant aux obligations prévues par la présente Convention, dans la stricte mesure où la situation l'exige et à la condition que ces mesures ne soient pas en contradiction avec les autres obligations découlant du droit international. 2. La disposition précédente n'autorise aucune dérogation à l'article 2 [droit à la vie], sauf pour le cas de décès résultant d'actes licites de guerre, et aux articles 3 [interdiction de la torture], 4 (paragraphe 1) et 7. » Voir également l'article 4, paragraphe 2, du Pacte international relatif aux droits civils et politiques de 1966 qui dispose notamment que l'article 7 n'est pas susceptible de dérogation. Voir aussi l'article 5 de la Convention interaméricaine contre la torture: « [n] e peut être invoquée ni admise comme justification du crime de torture l'existence de certaines circonstances, telles que l'état de guerre, la menace de guerre, l'état de siège, l'état d'alerte, les bouleversements ou conflits intérieurs, la suspension des garanties constitutionnelles, l'instabilité politique interne et d'autres crises ou calamités publiques. Le caractère dangereux du détenu ou du condamné, l’insécurité de la prison ou du pénitencier ne peuvent justifier la torture.»

34. Article 2, paragraphe 2, de la Convention. Au niveau régional, l'article 5 de la Convention interaméricaine pour la prévention et la répression de la torture s'inscrit dans cette droite ligne: «[n]e peut être invoquée ni admise comme justification du crime de torture l'existence de certaines circonstances, telles que l'état de guerre, la menace de guerre, l'état de siège, l'état d'alerte, les bouleversements ou conflits intérieurs, la suspension des garanties constitutionnelles, l'instabilité politique interne et d'autres crises ou calamités publiques. Le caractère dangereux du détenu ou du condamné, l'insécurité de la prison ou du pénitencier ne peuvent justifier la torture. »

35. Voir CEDH, Irlande c. Royaume-Uni, arrêt, 8 janvier 1978, série A, n²5, \$163; Chahal c. Royaume-Uni, req. n² 22414/93, arrêt, 15 novembre 1996, \$ 79; Selmouni c. France, arrêt, 28 juillet 1999, $\$ 95$; Al-Adsani c. Royaume-Uni, req. n 35763/97, arrêt (Grande Chambre), 21 novembre 2001, $\$ 59$; Chamaïev et autres c. Géorgie et Russie, req. $n^{\circ}$ 36378/02, arrêt, 12 avril 2005, \$335; Saadi c. Italie, req. n 37201/06, arrêt, 28 février $2008, \$ 127$.

36. CEDH, Chahal c. Royaume-Uni, req. $n^{\circ} 22414 / 93$, arrêt, 15 novembre 1996, $\$ 79$.

37. Ibid.

38. Voir les Lignes directrices du Comité des ministres du Conseil de l'Europe sur les Droits de l'homme et la lutte contre le terrorisme du 11 juillet 2002. Le point IV, intitulé «Interdiction absolue de la torture », est ainsi libellé : « [1] e recours à la torture ou à des peines ou traitements inhumains ou dégradants est prohibé en termes absolus, en toutes circonstances, notamment lors de l'arrestation, de l'interrogatoire et de la détention d'une personne soupçonnée d'activités terroristes ou condamnée pour de telles activités, et quels qu'aient été les agissements dont cette personne est soupçonnée ou pour lesquels elle a été condamnée. » Parallèlement, le point XV des Lignes directrices, intitulé «Dérogations éventuelles ", prévoit la possibilité pour les États d'adopter, lorsque la lutte contre le terrorisme intervient dans une situation de guerre ou de danger public qui menace la vie de la nation, des mesures dérogeant provisoirement à certaines obligations résultant des instruments internationaux de protection des Droits de l'homme. Le point XV énonce cependant, en son deuxième paragraphe, que «[1] es États ne peuvent toutefois, en aucun cas et quels qu'aient été les agissements de la personne soupçonnée d'activités terroristes, ou condamnée pour de telles activités, déroger au droit à la vie tel que garanti par ces instruments internationaux, à l'interdiction de la torture ou des peines ou traitements inhumains ou dégradants, au principe de la légalité des peines et mesures, ainsi qu'à celui de la rétroactivité pénale $»$. Nous soulignons.

39. Doc. ONU, A/RES/60/148, « Tortures et autres peines ou traitements cruels, inhumains ou dégradants », 21 février 2006. 
palestinien occupé $e^{40}$, la Cour internationale de justice a été amenée à statuer sur l'applicabilité des instruments internationaux concernant les Droits de l'homme auxquels Israël était partie dans le territoire palestinien. Alors qu'Israël faisait valoir que la protection de la population civile, en cas de conflit armé, relevait du seul droit international humanitaire, la Cour a mis en évidence l'application, en toutes circonstances, du droit international des Droits de l'homme ainsi que son application conjointe au droit humanitaire dans les situations de conflit armé ${ }^{41}$, ces deux branches du droit étant applicables à la même situation. Le Comité des Droits de l'homme de l'ONU a également considéré, lors de l'examen du Rapport des États-Unis, que le Pacte international relatif aux droits civils et politiques s'avérait applicable dans le cadre de la lutte contre le terrorisme, quand bien même les actions en question consistaient en des activités extraterritoriales ${ }^{42}$. Le Comité contre la torture a également interprété la portée dans l'espace de la Convention internationale contre la torture dans un sens similaire ${ }^{43}$.

Une lecture textuelle de la Convention contre la torture suggère que la clause dite de non-justification de la torture, c'est-à-dire le caractère absolu de l'interdiction en toutes circonstances, y compris dans le cadre de la lutte contre le terrorisme, ne s'applique qu'à la torture en tant que telle et n'embrasse pas la catégorie des traitements cruels, inhumains ou dégradants ${ }^{44}$, dont il n'est en effet pas fait mention à l'article 2 de la Convention. Contrairement à ce que la dénomination de la Convention suggère dans la mesure où son intitulé se réfère expressément aux traitements cruels, inhumains ou dégradants, il faut déplorer son silence s'agissant de cette notion qui ne se trouve pas définie plus avant et qui ne se trouve surtout pas expressis verbis concernée par les dispositions substantielles de la Convention. Celle-ci a principalement pour objet la torture en tant que telle, c'est-à-dire telle que définie à l'article $1^{\mathrm{er}}$. L'article 16 de la Convention énonce en effet que « [t] out État partie s'engage à interdire dans tout territoire sous sa juridiction d'autres actes constitutifs de peines ou traitements cruels, inhumains ou dégradants qui ne sont pas des actes de torture telle qu'elle est définie à l'article premier [...]». Il dispose ensuite que «les obligations énoncées aux articles 10, 11, 12 et 13 de la Convention sont, en particulier, applicables moyennant le remplacement de la mention de la torture par la mention d'autres formes de peines ou traitements cruels, inhumains ou dégradants ». Or, ces articles ne visent que l'obligation des États parties de veiller à ce que l'enseignement et l'information concernant l'interdiction de la torture fassent partie intégrante de la formation du personnel militaire et civil intervenant lors de l'arrestation ou de la détention d'individus; l'obligation de surveiller les règles et méthodes d'interrogatoire relativement au traitement des personnes arrêtées et détenues en vue d'éviter la torture; l'obligation de veiller à ce que les autorités compétentes procèdent à une enquête impartiale chaque fois qu'il y a des motifs de croire qu'un acte de torture a été commis et enfin l'obligation d'assurer à toute personne qui prétend avoir été soumise à la torture sur tout territoire soumis à sa juridiction le droit de porter plainte devant les autorités compétentes dudit État. Dès lors, certains États considèrent que si l'interdiction de la torture est absolue en toutes circonstances, ce n'est cependant pas le cas des «mauvais traitements» qui pourraient ainsi faire l'objet de dérogations en présence de circonstances exceptionnelles, à l'instar de la lutte contre le terrorisme.

Cette interprétation est cependant erronée et doit donc être réfutée. Les obligations énoncées à l'article 16 s'agissant des obligations des États parties ne sont en effet pas exhaustives comme en atteste l'adjectif «en particulier ». Surtout cette interprétation occulte le droit international coutumier ainsi que les interdictions des traitements cruels, inhumains ou dégradants qui figurent dans d'autres instruments internationaux, à l'instar des Conventions de Genève ou encore du Pacte international relatif aux droits civils et politiques de 1966 dont l'article 7 interdit expressément la torture et les autres peines ou traitements cruels, inhumains ou dégradants. Il faut en effet lire l'article 2 de la Convention en étroite

40. CIJ, Conséquences juridiques de l'édification d’un mur dans le territoire palestinien occupé, avis consultatif, 9 juillet 2004, CIJ Rec., 2004 , p. 136.

41. Ibid., $\$ 105$.

42. Voir les Observations du Comité des Droits de l'homme des Nations unies rendues publiques le 28 juillet 2006 formulées à l'occasion des $2^{\text {e }}$ et $3^{\text {e }}$ rapports périodiques présentés par les États-Unis sur la mise en œuvre par cet État des dispositions du Pacte international relatif aux droits civils et politiques.

43. Nations unies, Comité contre la torture, Convention contre la torture et autres peines ou traitements cruels, inhumains ou dégradants. Observation générale $\mathrm{n}^{\circ}$ 2, application de l'article 2 par les États parties, CAT/C/GC/2, 24 janvier 2008, $\$ 16$ : «le “territoire" s'étend à toutes les régions sur lesquelles l'État partie exerce de fait ou de droit, directement ou indirectement, en tout ou en partie, un contrôle effectif, conformément au droit international. Il considère que la référence à "tout territoire" [qui figure dans les dispositions pertinentes de la Convention] concerne les infractions commises non seulement à bord d'un navire ou d'un aéronef immatriculé sur le registre d'un État partie, mais aussi pendant une occupation militaire ou des opérations de maintien de la paix et dans des lieux tels qu'une ambassade, une base militaire, des locaux de détention ou tout autre espace sur lequel un État partie exerce un contrôle effectif. [...] La notion de "territoire" s'étend en outre aux situations dans lesquelles l'État partie exerce, directement ou indirectement, de fait ou de droit un contrôle sur les détenus. »

44. Sans entrer dans le détail de la définition des traitements cruels, inhumains ou dégradants, on se bornera à mentionner que les traitements cruels et inhumains impliquent des souffrances qui n'atteignent pas l'intensité de la torture ou dans lesquels l'un des éléments constitutifs de la torture fait défaut. Les traitements dégradants impliquent pour leur part l'humiliation de la victime ou des traitements qui sont disproportionnés par rapport aux circonstances des cas. Dans la mesure où la Convention contre la torture constitue le pivot central de la lutte internationale contre la torture, objet de cette étude, et compte tenu du cadre limité imparti à la présente étude, celle-ci n’envisage pas, si ce n’est de manière générale, la notion de traitements cruels, inhumains ou dégradants et se limite donc à l'examen de la torture. On se bornera à mentionner sur ce point la grande richesse de la jurisprudence de la Cour européenne des Droits de l'homme qui, par l'entremise d'une interprétation dynamique et évolutive de l'article 3 de la Convention, a progressivement favorisé l'extension de chacune de ces trois notions. Pour de plus amples développements sur cette notion, voir F. Sudre, "L'économie générale de l'article $3 \mathrm{CEDH}$ », in La portée de l'article 3 de la Convention européenne des Droits de l'homme, p. 9-11. 
liaison avec le paragraphe 2 de l'article 16 qui dispose que: «les dispositions de la présente Convention sont sans préjudice des dispositions de tout autre instrument international ou de la loi nationale qui interdisent les peines ou traitements cruels, inhumains ou dégradants. » En 2008, le Comité contre la torture a ainsi considéré que l'interdiction des mauvais traitements s'avérait, elle aussi, intangible en vertu de la Convention contre la torture, en raison notamment du lien ontologique qui unit les traitements cruels, inhumains ou dégradants et la torture. Il a, à ce propos, fait valoir que :

l'obligation de prévenir la torture consacrée à l'article 2 est de portée large. Cette obligation et celle de prévenir les traitements inhumains, cruels ou dégradants (ci-après « mauvais traitements »), énoncée au paragraphe 1, de l'article 16, sont indissociables, interdépendantes et intimement liées. Dans la pratique, l'obligation de prévenir les mauvais traitements recoupe celle d'empêcher que des actes de torture ne soient commis et lui est dans une large mesure équivalente. En identifiant les moyens de prévenir les mauvais traitements, l'article 16 met l'accent «en particulier» sur les mesures énoncées aux articles 10 à 13 , mais sans s'y limiter [...]. Dans la pratique, la ligne de démarcation entre les mauvais traitements et la torture est souvent floue. L'expérience montre que les circonstances qui sont à l'origine de mauvais traitements ouvrent souvent la voie à la torture; les mesures requises pour empêcher la torture doivent donc aussi s'appliquer à la prévention des mauvais traitements. C'est pourquoi le Comité a considéré que l'interdiction des mauvais traitements était elle aussi intangible en vertu de la Convention et que leur prévention devait être efficace et ne souffrir aucune exception $[\ldots]$ Le Comité considère que les obligations énoncées dans les articles 3 à 15 s'appliquent indifféremment à la torture et aux mauvais traitements ${ }^{45}$.

Les développements qui précèdent montrent la portée progressivement acquise par l'interdiction de la torture, son caractère absolu et non susceptible de suspension ou de dérogation venant souligner l'importance capitale du droit de ne pas être soumis à la torture ou à des peines ou traitements cruels, inhumains ou dégradants. Pour un certain nombre d'auteurs, l'interdiction formelle de toute dérogation à un droit conventionnellement consacré constitue en outre l'un des critères d'identification d'une norme relevant du droit impératif.

\section{Une interdiction relevant du jus cogens}

L'interdiction universelle de la torture a été considérée par un certain nombre d'auteurs et d'instances comme étant une norme désormais coutumière, même avant la conclusion de la Convention contre la torture ${ }^{46}$, relevant de la catégorie du jus cogens ${ }^{47}$, c'est-à-dire une norme de droit impératif qui lie tous les États et à laquelle il ne peut être dérogé en aucune circonstance ${ }^{48}$. Pour reprendre les termes de la Convention de Vienne sur le droit des traités de 1969 qui officialise pour la première fois la notion, une norme impérative du droit international général «est une norme acceptée et reconnue par la communauté internationale des États dans son ensemble en tant que norme à laquelle aucune dérogation n'est permise et qui ne peut être modifiée que par une nouvelle norme du droit international général ayant le même caractère ${ }^{49}$. En mentionnant ainsi l'existence de certaines normes

45. Nations unies, Comité contre la torture, Convention contre la torture et autres peines ou traitements cruels, inhumains ou dégradants. Observation générale $\mathrm{n}^{\circ}$ 2, application de l'article 2 par les États parties, CAT/C/GC/2, 24 janvier 2008, $\$ 3$.

46. Voir, en ce sens, J. H. Burgers et H. Danelius, The United Nations Convention against Torture..., p. 1. En 1980, une juridiction américaine parvint à la même conclusion dans l'affaire Filartiga v. Peña-Irala: «[t] urning to the act of torture, we have little difficulty discerning its universal renunciation in the modern usage and practice of nations. [...] [W] conclude that official torture is now prohibited by the law of nations. The prohibition is clear and unambiguous, and admits of no distinction between treatment of aliens and citizens. " Dolly M. E. Filartiga and Joel Filartiga v. Americo Norberto Peña-Irala, 630 F.2d 876 ( $2^{\mathrm{d}}$ Cir. 1980), p. 883-884; International Law Reports, vol. 77, p. 169-184. Pour conclure à la prohibition universelle de la torture au $\mathrm{XX}^{\mathrm{e}}$ siècle, le juge Kaufman se référa à de nombreux instruments internationaux tels que la Charte des Nations unies, la Déclaration universelle des Droits de l'homme, la Déclaration sur la protection de toutes les personnes contre la torture et autres peines ou traitements cruels, inhumains ou dégradants adoptée par l'Assemblée générale des Nations unies en 1975. Il s'appuya également sur l'interdiction de la torture figurant dans les Constitutions de plus de 55 États, comprenant tant le Paraguay que les États-Unis (Filartiga v. Peña-Irala, ibid., p. 881-885).

47. Voir l'Observation générale n² 24 du Comité des Droits de l'homme des Nations unies. Questions touchant les réserves formulées au moment de la ratification du Pacte ou des protocoles facultatifs y relatifs ou de l'adhésion à ces instruments, ou en rapport avec des déclarations formulées au titre de l'article 41 du Pacte, 4 novembre 1994, $\$ 10:$ : [...] certains droits auxquels il n’est pas permis de déroger - interdiction de la torture et de la privation arbitraire de la vie - [...] ne peuvent faire l'objet d'une réserve puisque ce sont des normes impératives. » Nous soulignons.

48. S'il peut exister un lien certain entre le caractère non dérogeable d'une norme et sa qualification en tant que norme de jus cogens, il n'existe pourtant pas d'identité parfaite entre ces deux notions qui doivent être distinguées. Si le caractère non dérogeable d'un droit et sa qualification de jus cogens peuvent en effet se recouper et se confondre pour souligner l'importance capitale du droit en question, comme en ce qui concerne l'interdiction de la torture, cela n'est cependant pas toujours le cas. Ainsi, certains droits, qui ne sont pas d'une importance capitale, n'en sont pas moins insusceptibles de dérogation tout simplement parce que « leur suspension est sans rapport avec le contrôle légitime de l'état d'urgence national (par exemple, l'interdiction de l'emprisonnement pour dettes faite à l'article 11 [du Pacte international relatif aux droits civils et politiques]. Une autre raison est que cette dérogation peut concrètement être impossible (comme, par exemple, dans le cas de la liberté de conscience) ». Observation générale n 24 du Comité des Droits de l'homme des Nations unies..., $\$ 10$. Voir encore l'Observation générale n 29 du Comité des Droits de l'homme des Nations unies sur l'article 4 du Pacte international relatif aux droits civils et politiques, 24 juillet 2001, CCPR/C/21/Rev.1/Add. 11, $\$ 11$ : «L'énumération des dispositions non susceptibles de dérogation figurant à l'article 4 du Pacte est liée - sans se confondre avec elle - à la question de savoir si certaines obligations relatives aux Droits de l'homme revêtent le caractère de norme impérative du droit international. Le fait que certaines dispositions du Pacte soient, au paragraphe 2 de l'article 4, proclamées non susceptibles de dérogation doit être interprété en partie comme une constatation du caractère impératif de quelques droits fondamentaux garantis par traité dans le Pacte (par exemple les articles 6 et 7). Il est évident toutefois que d'autres dispositions du Pacte ont été incluses dans la liste de celles auxquelles il ne peut être dérogé parce qu'elles portent sur des droits dont la dérogation ne peut jamais être rendue nécessaire par la proclamation d'un état d'exception. De plus, la catégorie des normes impératives est plus étendue que la liste des dispositions intangibles figurant au paragraphe 2 de l'article 4. "Sur la distinction entre normes indérogeables et normes relevant du jus cogens, voir J.-F. Flauss, «La protection des Droits de l'homme et les sources du droit international ", in La protection des Droits de l'homme et l'évolution du droit international, p. 75-78.

49. Article 53 de la Convention de Vienne sur le droit des traités du 23 mai 1969. 
non seulement obligatoires mais également impératives, en assignant un statut juridique supérieur à certaines valeurs fondamentales, la Convention de Vienne consacre l'existence d'intérêts supérieurs aux intérêts individuels des États. La notion de jus cogens introduit ce faisant l'idée d'une "normativité graduée ${ }^{50}$ au sein de l'ordre juridique international, une sorte de « hiérarchie» entre normes impératives du droit international général et les autres normes - cette «hiérarchie» étant avant tout d'ordre matériel à raison de l'importance des valeurs que les normes impératives protègent. La notion de jus cogens postule ainsi «l'existence d'une communauté juridique universelle fondée sur ses valeurs propres devant lesquelles tous ses membres doivent s'incliner ${ }^{51}$.

S'agissant des juridictions internationales, c'est le Tribunal pénal international pour l'ex-Yougoslavie (TPIY) qui a affirmé le premier, en 1998, la nature de jus cogens de l'interdiction de la torture. Le 16 novembre 1998, dans l'affaire Celebici, le TPIY rejeta l'argumentation de la défense selon laquelle la définition de la torture résultant de la Convention ne faisait pas partie du droit international coutumier, déclarant qu'il ne pouvait y avoir aucun doute sur l'interdiction de la torture tant en droit conventionnel qu'en droit coutumier. Au regard du corpus normatif existant, le Tribunal arriva à la conclusion selon laquelle « on peut dire que l'interdiction de la torture est une norme du droit coutumier. Elle constitue aussi une norme de jus cogens [...]. Il faut ajouter que l'interdiction édictée par les instruments internationaux susmentionnés est absolue et il ne peut y être dérogé en aucune circonstance ${ }^{52}$.

Quelques jours après la première décision de la Chambre des Lords relative à l'extradition du général Pinochet, la qualification de la prohibition de la torture en tant que norme de jus cogens a ensuite été réaffirmée, pour trouver sa formulation la plus aboutie, dans l'affaire Furundzija (1998). Le TPIY a considéré que l'interdiction de la torture constituait non seulement une règle obligatoire mais aussi une règle impérative relevant de la catégorie des normes de jus cogens ${ }^{53}$. Le Tribunal précise, à propos de l'interdiction de la torture, qu' « en raison de l'importance des valeurs qu'il protège, ce principe est devenu une norme impérative ou jus cogens, c'est-à-dire une norme qui se situe dans la hiérarchie internationale à un rang plus élevé que le droit conventionnel et même que les règles du droit coutumier "ordinaire”. La conséquence la plus manifeste en est que les États ne peuvent déroger à ce principe par le biais de traités internationaux, de coutumes locales ou spéciales ou même de règles coutumières générales qui n'ont pas la même valeur normative ${ }^{54}$.

Le Tribunal ajoute encore que: «[c]lairement, la valeur de jus cogens de l'interdiction de la torture rend compte de l'idée que celle-ci est désormais l'une des normes les plus fondamentales de la communauté internationale. En outre, cette interdiction doit avoir un effet de dissuasion en ce sens qu'elle rappelle à tous les membres de la communauté internationale et aux individus sur lesquels ils ont autorité qu'il s'agit là d'une valeur absolue que nul ne peut transgresser ${ }^{55}$.»

La jurisprudence du TPIY revêt une grande importance car c'est la première fois qu'un jugement international - même si celui-ci résulte d'une juridiction spécialisée de premier degré - qualifie une règle du droit international général de norme de jus cogens ${ }^{56}$. On relèvera ici, de manière incidente, que cette «audace» du TPIY contraste avec la grande réserve dont fait généralement preuve la Cour internationale de justice à l'égard de cette notion et particulièrement à l'égard de l'identification concrète des normes relevant du jus cogens ${ }^{57}$.

La Cour européenne des Droits de l'homme a ensuite pris acte de cette qualification et l'a reprise à son compte dans son arrêt rendu en 2001 dans l'affaire Al-Adsani c. Royaume-Uni ${ }^{58}$. Après avoir souligné, au regard des différents textes internationaux existants - au premier rang desquels elle mentionne l'article 5 de la Déclaration universelle des Droits de l'homme - que «l'importance primordiale que revêt l'interdiction de la torture est de plus en plus reconnue, comme en témoignent d'autres domaines du droit international ${ }^{59}$, la Cour s'est expressément fondée sur la jurisprudence du TPIY et sur le jugement de la Chambre des Lords rendu dans l'affaire Pinochet ${ }^{60}$

50. Selon l'expression de P. Weil, «Vers une normativité relative en droit international ?», RGDIP, n 1, 1982, p. 19.

51. V. Zakane, «La compétence universelle des États dans le droit international », African Yearbook of International Law, 2000, p. 190.

52. TPIY, Le Procureur c. Zejnil Delalic et autres (Celebici), aff. n IT-96-21-T, Chambre de première instance II quater, jugement, 16 novembre 1998 , $\$ 454$.

53. Cette qualification est depuis invariablement reprise dans la jurisprudence du TPIY. Voir notamment Le Procureur c. Dragoljub Kunarac, Radomir Kovac et Zoran Vukovic, aff. n IT-96-23-T \& IT-96-23/1-T, Chambre de première instance II, jugement, 22 février 2001, \$ 466: « [1] e droit international, tant conventionnel que coutumier, interdit la torture en temps de paix comme de conflit armé. On peut dire que cette prohibition a valeur de jus cogens.»

54. TPIY, Le Procureur c. Anto Furundzija, aff. nIT-95-17/1-T10, Chambre de première instance II, jugement, 10 décembre $1998, \$ 151$.

55. Ibid., $\$ 154$.

56. De manière générale, voir F. Harhoff, «La consécration de la notion de jus cogens dans la jurisprudence des Tribunaux pénaux internationaux », in Actualité de la jurisprudence pénale internationale à l'heure de la mise en place de la Cour pénale internationale, P. Tavernier (dir.), Bruxelles, Bruylant, 2004, p. 65-80.

57. La Cour a en effet longtemps contourné cette notion, pour ne l'évoquer que de manière indirecte. Ce n'est qu'en 2006, dans un arrêt rendu dans l'affaire des activités armées sur le territoire du Congo (nouvelle requête : 2002) (République démocratique du Congo c. Rwanda) que la Cour qualifie explicitement et pour la première fois l'interdiction du génocide de norme de jus cogens. (CIJ, Affaire des activités armées sur le territoire du Congo (nouvelle requête: 2002) (République démocratique du Congo c. Rwanda), Compétence de la Cour et recevabilité de la requête, arrêt, 3 février 2006 , $\$ 64$.

58. CEDH, Al-Adsani c. Royaume-Uni, req. n 35763/97, arrêt (Grande Chambre), 21 novembre 2001.

59. Ibid., $\$ 60$.

60. Dans l'affaire Pinochet soumise aux tribunaux britanniques, les Lords se sont en effet attachés à l'examen de la prohibition de la torture en droit international pour reconnaître que celle-ci avait probablement atteint la dimension d'une norme de jus cogens qui imposerait aux États des obligations erga omnes. Regina v. Bow Street Metropolitan Stipendiary Magistrate, ex parte Pinochet Ugarte ( ${ }^{\circ}{ }^{\circ}$ ), House of Lords, 24 mars 1999, International Law Reports, 
pour admettre, à son tour, «sur la foi de ces précédents jurisprudentiels, que l'interdiction de la torture est devenue une règle impérative du droit international $»^{61}$.

La Cour interaméricaine des Droits de l'homme a suivi une démarche similaire et ne manque pas de rappeler, dans sa jurisprudence, que l'article 5 de la Convention constitue une norme de jus cogens ${ }^{62}$.

La qualification d'une norme comme ressortissant au jus cogens suscite certaines critiques dont l'une a notamment trait à l'utilité même de ce "surcroît» de qualification en relation avec ses répercussions juridiques. Le jus cogens constitue en effet le caractère propre à certaines normes de ne pas être susceptibles de dérogation par voie conventionnelle. Consubstantielle au droit des traités, la notion de jus cogens signifie qu'une norme juridique qui relève de cette catégorie ne peut, en aucune circonstance, être écartée par voie d'accord entre deux ou plusieurs États. Le cas échéant, «est nul tout traité, qui, au moment de sa conclusion est en conflit avec une norme impérative du droit international général " ${ }^{63}$. Or, on imagine difficilement deux ou plusieurs États conclure un traité international pour violer délibérément une norme fondamentale de protection des Droits de l'homme. Au-delà de cette considération et des subtiles controverses auxquelles la notion donne lieu, le concept de jus cogens, appliqué à l'interdiction de la torture, présente un intérêt sur lequel il est, à notre avis, permis de s'accorder: «son intérêt est autre et se situe de lege ferenda dans la formation progressive du droit international. En dépassant le strict fondement volontariste du droit, l'affirmation que des normes coutumières relatives aux Droits de l'homme ont la qualité de normes impératives de jus cogens, parce que d'intérêt commun supérieur, participe à l'ancrage du respect des Droits de l'homme, comme élément constitutif d'un ordre public international en devenir ${ }^{64}$.»

En visant l'interdiction de comportements qui heurtent la conscience juridique universelle, la notion de jus cogens a en effet pour fonction d'assurer et de maintenir un degré minimum d'ordre public international.

La proclamation de l'interdit, aussi impérative soitelle, n'est cependant pas suffisante pour assurer son respect effectif.

\section{Le développement des mécanismes visant à prévenir la torture}

Depuis la proclamation de l'interdiction de la torture, différents mécanismes ont été établis en vue de faire respecter effectivement l'interdiction des pratiques de torture, tant au niveau international qu'au niveau régional. La prévention de la torture revêt à cet égard une importance capitale. La Convention des Nations unies contre la torture énonce à cet effet que tout État est tenu de prendre «des mesures législatives, administratives, judiciaires et autres mesures efficaces pour empêcher que des actes de torture ne soient commis ${ }^{65}$. Ces mesures sont diverses et comprennent l'interdiction sans équivoque de la torture mais également la formation du personnel de police et de sécurité, l'élaboration de normes précises concernant le traitement des personnes privées de liberté, la mise en place de mécanismes nationaux d'inspection et de supervision ou encore l'introduction d'un dispositif permettant d'enquêter efficacement sur les plaintes relatives à des mauvais traitements. Pour veiller au respect des principes qu'elle pose, la Convention contre la torture établit un Comité contre la torture, composé de dix experts indépendants, élus par les États parties à la Convention ${ }^{66}$. Tous les États parties sont tenus de présenter au Comité, à intervalles réguliers, des rapports sur les mesures qu'ils ont adoptées pour donner effet à leurs engagements en vertu de la Convention. Ils doivent ainsi présenter un premier rapport un an après avoir adhéré à la Convention, puis des rapports complémentaires tous les quatre ans sur les nouvelles mesures prises. Le Comité examine chaque rapport et fait part de ses préoccupations et recommandations à l'État partie sous la forme d'«observations finales " ${ }^{67}$. Le Comité peut, à sa discrétion, décider de reproduire tous les commentaires qu'il a formulés, accompagnés des observations qu'il a reçues à ce sujet de l'État partie, dans le rapport annuel qu'il établit. Pour s'acquitter de sa mission de surveillance de la mise en œuvre de la Convention par les États parties, le Comité peut également examiner des requêtes individuelles ou des communications émanant de particuliers qui prétendent être victimes d'une violation des droits reconnus par la Convention ${ }^{68}$, entreprendre des enquêtes ${ }^{69}$ et examiner les plaintes des États ${ }^{70}$.

vol. 119, p. 137-247. Voir les opinions de Lord Browne-Wilkinson, ILM, vol. 38, 1999, p. 589 ; de Lord Hutton, ibid., p. 637 ; de Lord Millet, ibid., p. 649 et de Lord Philipps of Worth Matravers, ibid., p. 661.

61. CEDH, Al-Adsani c. Royaume-Uni, $\$ 63$.

62. Voir CIDH, De la Cruz Flores c. Pérou, 18 novembre 2004, série C, n 115, \$125; Tibi c. Équateur, 7 septembre 2004, série C, $n^{\circ} 114$, $\$ 143$; Caesar c. Trinité et Tobago, 11 mars 2005 , série C, $\mathrm{n}^{\circ} 123, \$ 59$.

63. Article 53 de la Convention de Vienne sur le droit des traités.

64. F. Sudre, Droit international et européen des Droits de l'homme, $2^{\mathrm{e}}$ édition, Paris, PUF, 1995, p. 65.

65. Article 2, paragraphe 1, de la Convention.

66. Article 17 de la Convention.

67. Article 19 de la Convention.

68. Article 22 de la Convention. Les communications émanant de particuliers ne sont recevables que si l’État de la juridiction dont ils relèvent a reconnu la compétence du Comité pour examiner de telles communications.

69. Article 20 de la Convention.

70. Article 21 de la Convention. Là encore, la plainte d'un État relative à la pratique de la torture par un autre État n'est recevable que si le premier État a reconnu la compétence du Comité pour examiner des communications le concernant. 
Les actes de torture étant souvent commis dans des lieux isolés, les visites des lieux de détention s'avèrent fondamentales pour prévenir tout acte de torture. Ces visites permettent également de déceler les situations qui sont susceptibles d'entraîner des actes de torture et peuvent ainsi inciter les États à adopter les mesures appropriées pour réduire ce risque. L'idée d'organiser des visites régulières sur les lieux de détention remonte à la fin des années 1970. S’inspirant des visites effectuées par le Comité international de la Croix-Rouge en application des Conventions de Genève, Jean-Jacques Gautier, président du Comité suisse contre la torture, avait proposé l'adoption d'un nouvel instrument juridique contre la torture, doté de moyens efficaces de contrôle international des lieux privatifs de liberté ${ }^{71}$. Cette idée, sans doute alors trop ambitieuse pour pouvoir trouver à s'appliquer au niveau international, fut cependant retenue au niveau régional par le Conseil de l'Europe. La Convention européenne pour la prévention de la torture et des peines ou traitements inhumains ou dégradants, signée le 26 juin $1987^{72}$, entrée en vigueur en février 1989, ratifiée par tous les États membres du Conseil de l'Europe, établit un mécanisme supranational non judiciaire à caractère préventif qui consiste dans la création d'un Comité européen pour la prévention de la torture au sein du Conseil de l'Europe. Cet organe, composé d'experts indépendants, est chargé d'effectuer des visites périodiques ou ad hoc dans tous les lieux de détention relevant de la juridiction des États parties ${ }^{73}$. Le cas échéant, le Comité est habilité à effectuer des recommandations confidentielles au pays concerné ${ }^{74}$, afin d'améliorer certaines situations qui sont susceptibles d'entraîner la commission d'actes de torture. Les résultats de ces visites ont été jugés très positifs par les experts de la lutte contre la torture. Tous les États parties sont en outre tenus de présenter au Comité, à intervalles réguliers, des rapports sur la mise en œuvre des droits consacrés par la Convention.

Après cette adoption par le Conseil de l'Europe, le projet est revenu aux Nations unies, en 1992. À l'initiative du Costa Rica, la Commission des Droits de l'homme a établi un groupe de travail chargé d'examiner le projet de protocole facultatif à la Convention contre la torture. Les négociations devaient durer dix ans; elles aboutirent à l'adoption, par l'Assemblée générale des Nations unies, du protocole facultatif se rapportant à la Convention contre la torture et autres peines ou traitements cruels, inhumains ou dégradants, le 18 décembre $2002^{75}$. Il est entré en vigueur en juin $2006^{76}$. Le protocole repose sur le principe que des visites régulières aux lieux de détention constituent un moyen efficace pour prévenir la torture et les mauvais traitements. À la différence de la Convention européenne pour la prévention de la torture, le protocole présente la particularité - outre sa vocation universaliste - de mettre en place des visites régulières aux lieux de détention qui sont effectuées, de manière complémentaire, par un organe international, voisin du Comité européen - le Sous-Comité de la prévention ${ }^{77}$-, et par des experts indépendants propres à chaque État partie. Cela présente l'avantage de responsabiliser les autorités internes en matière de lutte contre la torture. En ratifiant ou en adhérant à ce protocole, les États parties acceptent des visites inopinées aux lieux de détention par ces organes. L'organe international - le Sous-Comité - se voit attribuer plusieurs tâches essentielles ${ }^{78}$. Sa mission principale consiste à effectuer des visites des lieux où se trouvent des personnes privées de liberté et à formuler, à l'issue de ces visites, des recommandations concernant la protection des personnes contre tout mauvais traitement à l'attention des États. De manière générale, les États parties sont tenus d'autoriser les membres du Sous-Comité à accéder aux lieux de détention qui se trouvent sur leur territoire; ils doivent aussi leur fournir toute information qu'ils demandent, faciliter les contacts entre le SousComité et les organismes nationaux de prévention, examiner les recommandations du Sous-Comité et adopter les mesures préconisées par celui-ci. Les recommandations sont transmises à l'État concerné, voire à son organisme national de prévention, à titre confidentiel ${ }^{79}$. La

71. Voir Comité Suisse contre la torture et Commission internationale des juristes, Torture: comment rendre efficace la Convention internationale (un projet de protocole facultatif), Lausanne, 1979.

72. Convention européenne pour la prévention de la torture et des peines ou traitements inhumains ou dégradants, Strasbourg, 26 juin 1987 , Série des traités européens, $\mathrm{n}^{\circ}$ 126. Sur cette Convention, voir A. Cassese, «A New Approach to Human Rights: The European Convention for the Prevention of Torture », AJIL, $\mathrm{n}^{\circ} 1,1989$, p. 128-153; E. Decaux, «La Convention européenne pour la prévention de la torture et des peines ou traitements inhumains ou dégradants », AFDI, 1988, p. 618-634.

73. Article 7 de la Convention.

74. Articles 10 et 11 de la Convention.

75. Voir doc. ONU, A/RES/57/199, 9 janvier 2003. Le protocole a été adopté par 127 voix pour, 42 abstentions et 4 voix contre. Sur ce protocole, voir N. Boeglin Naumovic et D. Long, Protocole facultatif contre la torture et autres peines ou traitements cruels, inhumains ou dégradants: un manuel pour la prévention, San José, Institut américain pour les Droits de l'homme, 2004; E. Delaplace, «Le protocole facultatif à la Convention des Nations unies contre la torture: un nouvel outil pour la prévention de la torture et des mauvais traitements ", Droits fondamentaux, $\mathrm{n}^{\circ} 4$, 2004: www.droits.fondamentaux.org, p. 133-140.

76. Conformément aux dispositions de son article 28 prévoyant son entrée en vigueur le trentième jour suivant le dépôt du vingtième instrument de ratification ou d'adhésion. Comme sa dénomination l'indique, le protocole est facultatif, ce qui permet aux États qui le souhaitent de prendre des engagements qui vont au-delà de ceux inclus dans la Convention de 1984. Tout État partie à la Convention onusienne contre la torture peut y adhérer, sans que cela constitue une obligation. Au 5 janvier 2009, 41 États étaient parties à ce protocole.

77. Selon l'article 5 du protocole, le Comité comporte actuellement 10 membres, traduisant une répartition géographique équitable (ceux-ci sont originaires d'Argentine, du Costa Rica, de Croatie, du Danemark, de l’Espagne, du Mexique, de l'Uruguay, de la Pologne, de la République tchèque, du Royaume-Uni) ; ce nombre sera porté à 25 lorsque le nombre d'États parties au protocole aura atteint 50.

78. Celles-ci sont décrites à l'article 11 du protocole.

79. Article 16 du protocole. 
publication du rapport concernant un État n'est en général possible qu'avec son assentiment. Il peut toutefois être publié sans son accord à titre de sanction des manquements de l'État aux engagements qu'il a contractés ou si l'État en question refuse d'adopter les mesures préconisées par le Sous-Comité. Des conditions préalables doivent cependant être réunies: le Sous-Comité doit demander au Comité contre la torture de décider de cette publication, à la majorité de ses membres, après que l'État concerné ait eu l'occasion de s'expliquer. Il est probable que ce mécanisme de sanction ne sera mis en œuvre qu'en cas de manquements manifestes et répétés de la Convention, ce qui prive dès l'abord le protocole d'une partie de son efficacité.

La véritable innovation du protocole par rapport au mécanisme européen de prévention de la torture réside en fait dans l'obligation qu'il établit à destination des États parties de mettre en place un mécanisme national de prévention ${ }^{80}$ qui se voit doter de missions similaires à celles du Sous-Comité, à savoir : examiner régulièrement la situation des personnes privées de liberté; formuler des recommandations à destination des autorités compétentes en vue d'améliorer le traitement et la situation de ces personnes et de prévenir tout mauvais traitement; formuler des propositions ou des observations sur la législation en vigueur ou les projets de loi en la matière ${ }^{81}$. C'est dans cette articulation entre un Sous-Comité de la prévention à vocation universelle et des mécanismes nationaux de prévention dont chaque État partie est tenu de se doter ${ }^{82}$ que réside la nouveauté du protocole.

L'existence de différents mécanismes de contrôle du respect par les États parties de leurs obligations conventionnelles qu'il s'agisse de la technique des rapports, établis par les autorités gouvernementales appelées à donner les raisons de la divergence de leur législation interne avec les dispositions de la Convention contre la torture, des visites sur les lieux de détention, de l'examen des requêtes individuelles des particuliers estimant avoir été victimes d'actes de torture ou encore des plaintes des États, s'avère à même d'inciter les États à s'acquitter plus rigoureusement de leurs obligations. Les observations et commentaires sur les mesures prises par les États par les différents comités permettent d'attirer l'attention des gouvernements mis en cause et de les appeler à un respect scrupuleux de leurs obligations, ceux-ci pouvant par exemple subséquemment modifier leur législation ou leur pratique de manière à ce qu'elles soient en conformité avec les exigences de la Convention. Pour autant, il faut rester conscient de la portée limitée des différentes procédures instituées qui ne disposent finalement pas de moyens de contraindre l'État à se conformer à ses obligations ou à mettre un terme aux violations constatées des droits : elles demeurent en effet tributaires de la bonne volonté de l'État souverain. Plus avant, l'efficacité de ces différentes procédures se heurte bien souvent à la résistance de nombreux États à toute forme de contrôle international, aussi souple soit-il. Le principal problème en la matière demeure ainsi la reconnaissance pleine et entière par les États parties de la compétence des Comités institués pour examiner les communications des particuliers ou les plaintes des États parties ${ }^{83}$.

\section{De la répression à la réparation de la torture}

L'obligation de poursuivre les auteurs présumés responsables d'actes de torture fait partie des obligations positives placées à la charge de l'État. La répression relève en tout premier lieu de la justice interne. Mais, au-delà des hypothèses classiques dans lesquelles un État peut exercer sa compétence juridictionnelle en la matière, qu'il s'agisse de la compétence territoriale ou de la compétence personnelle, liée à la nationalité de l'auteur présumé de l'infraction (compétence personnelle active) ou à celle de sa victime (compétence personnelle passive), la Convention internationale contre la torture présente une dimension supplémentaire qui consiste en l'universalisation de la répression des tortionnaires (A). En complément de la répression des auteurs présumés responsables d'actes de torture, la Convention internationale contre la torture établit en outre un véritable « droit à réparation » au profit des victimes d'actes de torture (B).

\section{A. L'universalisation de la répression de la torture}

Au-delà de la consécration conventionnelle de la compétence universelle en matière de torture (1), un certain

80. L'article 17 du protocole accorde aux États parties un délai d'un an pour «administrer, désigner ou mettre en place [...] un ou plusieurs mécanismes nationaux de prévention indépendants en vue de prévenir la torture à l'échelon national».

81. Article 19 du protocole.

82. On relèvera que la France entend pleinement participer à ce processus. Après avoir signé le protocole le 16 septembre 2005 , elle a en effet institué un contrôleur général des lieux de privation de liberté, au titre du mécanisme national de prévention de la torture prévu par le protocole (Loi n²007-1545 du 30 octobre 2007 instituant un contrôleur général des lieux de privation de liberté) qu’elle a finalement ratifié le 28 juillet 2008 . Pour un examen (critique) de la conformité de ce mécanisme de prévention de la torture avec les dispositions du protocole, voir le Rapport fait au nom de la Commission des affaires étrangères sur le Projet de loi, adopté par le Sénat, autorisant l'approbation du protocole facultatif se rapportant à la Convention contre la torture et autres peines ou traitements cruels, inhumains ou dégradants par M. Jean Glavany, II, B), 2. Document de l'Assemblée nationale, rapport $\mathrm{n}^{\circ}$ 1044, enregistré à la Présidence de l'Assemblée nationale le 9 juillet 2008.

83. En ce sens, voir la résolution 60/148 de l’Assemblée générale des Nations unies qui «invite tous les États parties qui ne l’ont pas encore fait à faire les déclarations prévues aux articles 21 et 22 de la Convention, relatifs aux communications entre États et aux communications émanant de particuliers, à envisager de retirer leurs réserves à l'article 20 [cet article prévoit la compétence du Comité contre la torture pour procéder à une enquête dès lors qu'il reçoit des renseignements crédibles qui lui semblent contenir des indications bien fondées que la torture est pratiquée systématiquement sur le territoire d'un État partie, auquel il transmet ces conclusions, commentaires et suggestions qu'il juge appropriés, celles-ci demeurant confidentielles] ». Doc. ONU, A/RES/60/148, 16 décembre 2005, $\$ 14$. 
courant de pensée fait valoir que c'est le caractère impératif de l'interdiction de la torture qui confère ipso facto et ipso jure à tout État un titre juridique à exercer la compétence universelle. Il convient donc de tenter de clarifier le lien existant entre les normes fondamentales et la compétence universelle (2) avant d'examiner la question de sa mise en ouvre effective par les États (3).

\section{La consécration conventionnelle de la compétence universelle à l'égard de la torture}

La torture suscite désormais une réprobation universelle qui se trouve assortie d'un mécanisme de compétence universelle des États parties à la Convention des Nations unies contre la torture pour poursuivre, et le cas échéant, juger et condamner les tortionnaires. La compétence universelle donne vocation à juger une infraction internationale considérée comme particulièrement grave aux tribunaux de l'État sur le territoire duquel son auteur est arrêté ou se trouve même passagèrement, alors même que cette infraction n'a pas été commise sur son territoire, que la victime et l'auteur de cette infraction ne sont pas ses ressortissants et que cette infraction n'a pas porté atteinte à ses intérêts. À la différence des chefs traditionnels de compétence pénale qui ont pour but principal de protéger les intérêts nationaux, la compétence universelle se réfère ainsi à la protection de valeurs universellement reconnues et repose sur l'idée de solidarité des États dans la répression des infractions internationales qui portent atteinte à certains intérêts fondamentaux de la communauté internationale. Cette technique juridique de répression pénale répond ainsi parfaitement à l'idée selon laquelle à la prohibition universelle du crime doit répondre l'universalisation de la répression. En s'assurant, notamment en cas de défaillance de la justice nationale, qu'un juge sera toujours compétent pour connaître des faits de torture, quel que soit l'État sur le territoire duquel l'auteur de l'infraction aura trouvé refuge, ce mécanisme permet en effet d'assurer l'ubiquité de la répression et, en dernier ressort, sa certitude.

C'est dans ce cadre que la Convention internationale contre la torture fait obligation aux États parties d'inscrire le principe de la compétence universelle dans leur ordre juridique interne. Celle-ci est envisagée par l'entremise de l'alternative aut dedere aut judicare (soit extrader, soit juger) en vertu de laquelle l'État partie sur le territoire duquel se trouve l'auteur présumé responsable d'un acte de torture doit, soit l'extrader, soit le juger luimême ${ }^{84}$. Parallèlement, la Convention dispose ensuite, en son article 7, que : «1. [1]'État partie sur le territoire sous la juridiction duquel l'auteur présumé d'une infraction visée à l'article 4 est découvert, s'il n'extrade pas ce dernier, soumet l'affaire, dans les cas visés à l'article 5, à ses autorités compétentes pour l'exercice de l'action pénale. »

Cet article établit ainsi une obligation d'exercice de la compétence universelle alternative (à l'extradition). La mise en œuvre de cette obligation rencontre théoriquement la liberté des États dans la détermination de leur choix entre les deux options considérées. L'initiative de l'insertion de cette disposition revient à la Suède. La proposition suédoise partait du postulat que si l'État sur le territoire duquel les actes de torture avaient été commis procèderait certes à la répression de leurs auteurs lorsque ceux-ci étaient des acteurs privés, il ne procèderait pas, en revanche, à leur répression dès lors que les actes en question auraient été commis par des agents étatiques.

Si le mécanisme juridictionnel retenu trouve son origine dans les traités internationaux des années 1970 visant la répression du terrorisme ${ }^{85}$, il s'avère cependant plus inhabituel en matière de protection des Droits de l'homme. À la différence des infractions de terrorisme qui sont clairement marquées par leur dimension transnationale et qui correspondent à une criminalité mouvante et insaisissable, les infractions relatives aux Droits de l'homme se caractérisent en effet par le fait qu'elles peuvent être commises à l'intérieur des frontières d'un seul État. Dès lors, on comprend aisément que le principe de souveraineté de l'État et son corollaire, le principe de non-ingérence dans les affaires intérieures, aient longtemps fait obstacle à toute extension de la portée extraterritoriale de la répression, la compétence universelle ayant notamment été refusée par les États lors de la conclusion de la Convention sur le génocide, en 1948. La Convention contre la torture parachève ainsi le processus de consécration conventionnelle de la compétence universelle en matière de violations des Droits de l'homme $^{86}$. Cette évolution s'explique par le mouvement irrémédiable d'internationalisation des Droits de l'homme ${ }^{87}$ et par l'idée selon laquelle le traitement de ses nationaux par un État ne constitue plus une question relevant du ressort de ses affaires intérieures.

84. Article 5, paragraphe 2, de la Convention.

85. Voir la Convention de La Haye du 16 décembre 1970 pour la répression de la capture illicite d'aéronefs (art. 4, paragraphes 2 et 7 de la Convention).

86. Si la Convention internationale sur l'élimination et la répression du crime d'apartheid de 1973 établit pour la première fois en matière de Droits de l'homme le principe de la compétence universelle, l'approche retenue demeure cependant extrêmement prudente. Si la Convention établit en effet, à la charge des États parties, une obligation d'établir la compétence universelle dans leur ordre juridique interne, celle-ci ne se trouve assortie que d'une simple faculté d'exercice (art. V de la Convention), à la différence de la Convention contre la torture qui établit une obligation d'exercice de la compétence universelle. Dans un souci de référencement exhaustif des mécanismes conventionnels existants s'agissant de la répression de la torture prévoyant la compétence universelle, il faut relever que la convention onusienne susmentionnée a directement inspiré la conclusion, au niveau régional, de la Convention interaméricaine pour la prévention et la répression de la torture, signée le 9 décembre 1985. La Convention établit un dispositif répressif qui prévoit expressément le principe aut dedere aut judicare, dont la formulation semble cependant placer au premier plan l'obligation d'extrader à la charge de l'État du lieu d'arrestation du prévenu, la compétence universelle retenue devant ainsi être envisagée par l'entremise de la formulation primo dedere, secundo judicare (compétence universelle obligatoire mais subsidiaire).

87. Sur ce point, voir G. Cohen-Jonathan, «L'évolution du droit international des Droits de l'homme», Mélanges offerts à Hubert Thierry: l'évolution du droit international, Paris, Pedone, 1998, p. 107-125. 


\section{Le lien existant entre les normes fondamentales et la compétence universelle}

L'interdiction universelle de la torture, nous l'avons vu, est considérée comme une norme de jus cogens. Or, pour un certain nombre d'auteurs, cette qualification se trouve assortie de l'application consubstantielle de la compétence universelle. Il existerait une règle coutumière conférant une compétence universelle à tout État dès lors que le crime à l'égard duquel elle est invoquée est susceptible d'être qualifié de crime de droit international ou de violation du jus cogens. C'est notamment en ce sens que se prononça Lord Millet dans l'affaire Pinochet, dans l'arrêt de la Chambre des Lords rendu le 24 mars 1999, considérant que la compétence universelle à l'égard de la torture existait avant sa consécration conventionnelle de 1984: «[i]n my opinion, crimes prohibited by international law attract universal jurisdiction under customary international law if two criteria are satisfied. First, they must be contrary to a peremptory norm of international law so as to infringe a jus cogens. Secondly, they must be so serious and on such a scale that they can justly be regarded as an attack on the international legal order. Isolated offences, even if committed by public officials, would not satisfy these criteria $[. .$.$] . Every State has jurisdiction under customary$ international law to exercise extraterritorial jurisdiction in respect of international crimes which satisfy the relevant criteria. In my opinion, the systematic use of torture on a large scale and as an instrument of state policy had joined piracy, war crimes and crimes against peace as an international crime of universal jurisdiction well before $1984^{88}$. "

C'est cependant au TPIY que l'on doit d'avoir le plus clairement mis en évidence le lien entre les normes impératives et la compétence universelle ${ }^{89}$ dans l'affaire Furundzija. Le Tribunal établit en effet un lien direct entre «la valeur de jus cogens reconnue à l'interdiction de la torture par la communauté internationale ${ }^{90}$ et la reconnaissance de la compétence universelle qui est présentée comme la conséquence logique du caractère impératif de la norme violée. Selon le Tribunal : «[...] il semblerait que l'une des conséquences de la valeur de jus cogens reconnue à l'interdiction de la torture par la communauté internationale fait que tout État est en droit d'enquêter, de poursuivre et de punir ou d'extrader les individus accusés de torture, présents sur son territoire. En effet, il serait contradictoire, d'une part, de restreindre, en interdisant la torture, le pouvoir absolu qu'ont normalement les États souverains de conclure des traités et, d'autre part, d'empêcher les États de poursuivre et de punir ceux qui la pratiquent à l'étranger. Ce fondement juridique de la compétence universelle des États en matière de torture confirme et renforce celui qui, de l'avis d'autres juridictions, découle du caractère par essence universel du crime. On a estimé que les crimes internationaux étant universellement condamnés quel que soit l'endroit où ils ont été commis, chaque État a le droit de poursuivre et de punir les auteurs de ces crimes. Comme le dit de façon générale la Cour suprême d'Israël dans l'affaire Eichmann, de même qu'une juridiction des États-Unis dans l'affaire Demjanjuk, 'c'est le caractère universel des crimes en question” (c'est-à-dire des crimes internationaux) qui confère à chaque État le pouvoir de traduire en justice et de punir ceux qui y ont pris part ${ }^{91}$.

Pour certains auteurs, «[i]l n'est pas interdit de voir dans l'arrêt Furundzija un précédent de première importance dans le développement jurisprudentiel du droit international pénal. Sa portée n'est pas mince. Elle ouvre la voie à la désignation des "crimes les plus graves qui touchent l'ensemble de cette branche du droit qui serait concernée par les implications, substantielles mais également procédurales, d'une telle qualification (dont l'une est que la poursuite de tels crimes relève logiquement de la compétence universelle) ${ }^{92}$.

Pour autant, une lecture attentive de l'obiter dictum du jugement du TPIY invite à la prudence. Elle permet en effet de réduire singulièrement la portée de la création du Tribunal, liant le caractère impératif de la prohibition de la torture, ainsi que sa qualification en tant que crime de droit international universellement condamné, à l'exercice de la compétence universelle. En premier lieu, on peut être surpris par la conséquence attachée par le Tribunal au caractère impératif de l'interdiction de la torture qui n'est que celle d'autoriser tout État à exercer la compétence universelle alors qu'on aurait légitimement pu s'attendre à une obligation d'exercer la compétence universelle compte tenu de la qualification attachée à

88. Opinion de Lord Millet, ILR, vol. 119, p. 229-230. Le juge poursuivit son argumentation en considérant que la Convention de 1984 « [...] affirmed and extended an existing international crime and imposed obligations on the parties to the Convention to take measures to prevent it and to punish those guilty of it. [...] Whereas previously states were entitled to take jurisdiction in respect of the offence wherever it was committed, they were now placed under an obligation to do so ». Opinion de Lord Millet, ILR, vol. 119, p. 230-231.

89. Il faut également mentionner, au niveau interne, une ordonnance rendue par un juge d'instruction belge faisant suite à une plainte avec constitution de partie civile déposée le $1^{\text {er }}$ novembre 1998 par des ressortissants chiliens entre les mains du juge d'instruction bruxellois contre Augusto Pinochet. Par une ordonnance du 6 novembre 1998, le magistrat s'est en effet reconnu compétent pour connaître des poursuites intentées à l'encontre de l'ancien chef d'État qui était alors détenu à Londres. Cette ordonnance avait pour objet la demande d'un acte d'instruction complémentaire en vue de l'extradition du général Pinochet pour qu'il soit jugé par les tribunaux belges, en vertu de la compétence universelle. Pour fonder sa compétence, le juge évoque le jus cogens comme « autorisant les autorités étatiques nationales à poursuivre et à traduire en justice, en toutes circonstances, les personnes soupçonnées de crimes contre l'humanité ». Voir Tribunal de première instance de l'arrondissement de Bruxelles. Ordonnance du Juge d'instruction Damien Vandermeersch, Article 61 quinquies C.I.Cr, demande d'un acte d'instruction complémentaire, 6 novembre 1998, point 3.3.3. de l'ordonnance, $Y I H L, 1999$, p. 475-485. Pour une analyse critique, voir J. Verhoeven, «M. Pinochet, la coutume internationale et la compétence universelle", Journal des Tribunaux, 1999, p. 308-315.

90. TPIY, Le Procureur c. Anto Furundzija, \$154.

91. Ibid., \$156. Nous soulignons.

92. P.-M. Dupuy, «Normes internationales pénales et droit impératif», in Droit international pénal, H. Ascensio, E. Decaux et A. Pellet (éd.), Paris, Pedone, 2000, p. 80. 
l'interdiction de la torture. En second lieu, le Tribunal n'affirme pas expressément, comme on l'a écrit ${ }^{93}$, que le caractère impératif de l'interdiction de la torture constitue ipso facto et ipso jure le fondement de l'exercice de la compétence universelle. Le Tribunal se borne en effet à déclarer que ce caractère impératif ne fait que « confirmer et renforcer» le fondement juridique de la compétence universelle qui préexiste donc à la qualification de la norme. La qualification de l'interdiction de la torture en tant que norme de jus cogens ne constitue ainsi qu'un argument supplémentaire propre à légitimer, de manière surabondante, l'exercice de la compétence universelle: « de l'aveu même du TPIY, il n'y a pas de corrélation immédiate entre le caractère impératif d'une prohibition et la compétence universelle pour en punir les contrevenants, puisqu'il ne s'agit que d'un fondement juridique supplémentaire $[\ldots]$. Le caractère fondamental d'une norme, dont le jus cogens est le degré ultime, n'aurait donc d'autre fonction que d'en quelque sorte "doper" la justification de l'établissement d'une compétence universelle ${ }^{94}$. "

Le Tribunal délaisse d'ailleurs cette considération et se cantonne ensuite à relever que l'«on a estimé» que le caractère par essence universel du crime entraînait le droit pour chaque État d'en poursuivre et d'en punir les auteurs.

À l'analyse, il convient en fait de remettre en cause le bien-fondé de la théorie en vertu de laquelle la compétence universelle s'avérerait juridiquement consubstantielle au caractère de jus cogens de l'interdiction de l'infraction.

Tout d'abord, il paraît difficile de justifier la compétence universelle par référence à la seule qualification de "crime de jus cogens» en raison de la nature même de l'institution du jus cogens. Selon les dispositions de la Convention de Vienne, la conséquence pratique de la qualification d'un crime comme relevant du jus cogens est stricto sensu la nullité absolue d'accords internationaux prévoyant la commission de tels crimes, même si ce n'est là qu'une hypothèse d'école. D'une institution destinée à déterminer la validité des rapports entre États, la notion de jus cogens apparaît ainsi singulièrement détournée de sa fonction originelle et substantielle. On lui attache en effet des conséquences qu'elle ne comporte pas, comme celle de constituer un mécanisme qui impose des obligations, non seulement aux États, mais également et surtout, dans le cadre de nos propos, aux individus. Or, la question de l'opposabilité des normes internationales du jus cogens aux individus demeure posée. Il est clair qu'à l'origine, le concept n'a été envisagé qu'à l'égard des États ou sujets de droit international qui étaient en mesure de se lier par des accords internationaux, et donc nullement aux individus. Dans sa seconde extension, le jus cogens est utilisé comme un moyen de preuve de l'existence d'une règle de droit, celle de l'existence de la compétence universelle à l'égard des crimes qualifiés comme tels. Pourtant, la question de la répression pénale de comportements individuels devant les juridictions nationales semble totalement étrangère à l'objet même de la notion de jus cogens. Il paraît difficile de mêler des branches de droit bien distinctes et donc d'admettre que l'interdiction d'un crime de droit international en tant que norme de jus cogens signifie que les États admettent la poursuite et le jugement universels de ces crimes. L'absence de correspondance directe entre la notion de jus cogens et la compétence universelle a été mise en exergue par l'Institut de droit international lors de son examen de la compétence universelle. Les propos de Mohamed Bennouna sont à cet égard représentatifs des vues de l'Institut: «[j]e ne vois pas pourquoi on confondrait les normes de jus cogens avec la juridiction universelle. Il s'agit de deux concepts qui poursuivent des objectifs complètement différents, le premier est en rapport avec les limitations à la capacité de contracter des États et le second concerne la poursuite au niveau universel d'infractions d'une gravité telle qu'elles mettent en cause certaines valeurs fondamentales de la communauté internationale. Bien entendu, il peut y avoir des interférences; la juridiction universelle concerne des infractions à certaines normes de droit impératif. Mais la réciproque est loin d'être vraie dans la mesure où toutes les infractions à des normes de jus cogens ne relèvent pas de la juridiction universelle ${ }^{95}$. »

Enfin, il faut relever que la justification de la compétence universelle par le recours à la notion de jus cogens ne se trouve pas corroborée par la pratique des États ${ }^{96}$ qui, tout en se référant au caractère universel de l'intérêt lésé par un crime de droit international, sur sa nature de crime dont l'interdiction relève du jus cogens, se fondent la plupart du temps sur une convention internationale pour justifier juridiquement l'exercice de la compétence universelle. S'agissant précisément de la torture, la position adoptée par la Chambre des Lords dans l'affaire Pinochet illustre à point nommé les dissonances de la pratique étatique par rapport à la construction du TPIY, établissant une correspondance directe entre la compétence universelle et le caractère de norme de jus cogens attribué à l'interdiction de la torture. Si les Lords se sont en effet accordés pour reconnaître sans ambages que la torture constituait un crime de droit international dont

93. Voir A. Bianchi, «Immunity versus Human Rights: The Pinochet Case», European Journal of International Law, n 2, 1999 , p. 262 ou P.-M. Dupuy, «Normes internationales pénales et droit impératif », p. 80. Contra, voir M. Cosnard, «La compétence universelle en matière pénale», in The Fundamental Rules of the International Legal Order: Jus Cogens and Obligations Erga Omnes, C. Tomuschat et J.-M. Thouvenin (éd.), Leyde, Nijhoff, 2006, p. 355-372 (359-364).

94. M. Cosnard, «La compétence universelle en matière pénale», p. 361.

95. Institut de droit international, «La compétence universelle en matière pénale à l'égard du crime de génocide, des crimes contre l'humanité et des crimes de guerre ", Annuaire de l’Institut de droit international, vol. 71, t. I, $\$$ 4, p. 278. Pour une position identique, voir M. Y. Dinstein, ibid., $\$ 4$, p. 267 : "[s] ince the scope of jus cogens is debatable, I am opposed to any attempt to use sweeping statements about an automatic link between jus cogens and universal jurisdiction. In any event, it cannot be ignored that the 1948 Genocide Convention rejected the application of universal jurisdiction, although genocide is an incontestable example of jus cogens. »

96. Pour un recensement détaillé de la pratique étatique, voir I. Moulier, La compétence pénale universelle en droit international, thèse, Université Paris IPanthéon-Sorbonne, 2006, Partie I, Chap. II, Section II, \$2. 
l'interdiction revêtait, même avant 1984 - date de conclusion de la Convention contre la torture -, le caractère de norme de jus cogens ${ }^{97}$, ils n'ont cependant pas considéré que la qualité de règle impérative de l'interdiction de la torture octroyait en elle-même une compétence universelle aux juridictions britanniques pour poursuivre l'ancien chef d'État pour des faits qui n'avaient aucun lien de rattachement avec le Royaume-Uni. Tout en reconnaissant le caractère de jus cogens à l'interdiction de la torture, ils n'en ont cependant tiré aucune implication directe et immédiate en matière de reconnaissance de la compétence universelle, qui découlerait de cette seule classification. Lord Phillips of Worth Matravers fit ainsi valoir: " it is still an open question whether international law recognises universal jurisdiction in respect of interna-

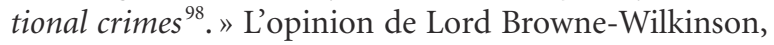
qui a rallié l'opinion du plus grand nombre sur le dispositif du jugement, présente à cet égard un intérêt particulier parce qu'elle s'appuie sur l'arrêt du TPIY rendu en l'affaire Furundzija. Le juge considéra que l'appartenance de l'interdiction de la torture au droit impératif ne justifiait pas, à elle seule, la reconnaissance de la compétence universelle avant l'entrée en vigueur de la Convention contre la torture au Royaume-Uni : « [a]t that stage, there was no international tribunal to punish torture and no general jurisdiction to permit or require its punishment in domestic courts. Not until there was some form of universal jurisdiction for the punishment of the crime of torture could it really be talked about as a fully constituted international crime. But in my judgment the Torture Convention did provide what was missing: a worldwide universal jurisdiction ${ }^{99}$.»

Au final, la Chambre des Lords s'en est tenue à une stricte application des dispositions conventionnelles résultant de la Convention contre la torture telles qu'incorporées en droit interne, considérant que le crime de torture pouvait être poursuivi en Grande-Bretagne sur le fondement de la compétence universelle à compter du 29 septembre 1988, date à laquelle la Convention contre la torture avait été incorporée en droit britannique. En se référant ainsi à la compétence universelle telle que prévue par le droit britannique et non par le droit international, la Chambre des Lords a implicitement réfuté la compétence universelle coutumière. Si les juges avaient en effet admis que le seul statut de règle impérative de l'interdiction de la torture suffisait à fonder la compétence universelle, ils ne se seraient en effet pas référés à la date d'introduction de la Convention contre la torture dans leur ordre juridique interne. L'arrêt Pinochet s'inscrit ainsi à contre-courant de l'argumentation en faveur de la reconnaissance large de la compétence universelle qui avait été opérée par le TPIY.

En fait, il n'est pas certain que la justification de la compétence universelle par le recours à la notion de jus cogens soit réellement nécessaire, si ce n'est pour souligner l'importance particulière des valeurs qui sont mises en cause par la commission de l'infraction, le jus cogens étant de fait utilisé comme un argument d'autorité morale supplémentaire au soutien de la seule légitimité de la compétence universelle à l'égard de certaines infractions sans que celle-ci soit pour autant justifiée juridiquement.

En revanche, la consécration de l'interdiction d'un comportement comme ressortissant au jus cogens pourrait emporter des conséquences directes au niveau de la mise en œuvre concrète de la compétence universelle dès lors que celle-ci est reconnue conventionnellement, comme c'est le cas pour la torture. On peut à cet égard soutenir, avec Michel Cosnard, que l'opposabilité universelle de l'incrimination entraîne l'opposabilité universelle des autorisations conventionnelles d'exercer la compétence universelle pour en poursuivre les auteurs puisque la notion de jus cogens aboutit à un affranchissement des règles traditionnelles qui régissent le droit des traités, et, de manière plus générale, des mécanismes de réciprocité $^{100}$. Ainsi, lorsqu'une convention établit la compétence universelle à l'égard d'un crime dont l'interdiction relève du jus cogens, le principe de l'effet relatif des traités ne saurait être invoqué si l'État territorial ou national n'est pas partie à la Convention. Cette question se trouve d'ailleurs concrètement posée à la Cour internationale de justice dans l'affaire relative à Certaines procédures pénales engagées en France, dite l'affaire des Disparus $d u$ Beach, qui oppose la République démocratique du Congo à la France. En l'espèce, la République du Congo a introduit, le 9 décembre 2002, une requête contre la France devant la Cour. Cette requête vise à faire annuler les actes d'instruction et de poursuite accomplis par la justice française à la suite d'une plainte émanant de diverses associations mettant en cause le président de la République du Congo, le ministre congolais de l'Intérieur ainsi que d'autres personnes, pour crimes contre l'humanité et tortures, commis au Congo contre des personnes de nationalité congolaise. Dans sa requête introductive d'instance, le Congo fait notamment valoir que les dispositions de la Convention des Nations unies contre la torture de 1984, en vertu de laquelle la France entend exercer sa compétence universelle, ne sauraient lui être opposées dans la mesure où il n'est pas partie à cette Convention ${ }^{101}$.

97. Lord Browne-Wilkinson, ILM, vol. 38, 1999, p. 589 ; Lord Hutton, ibid., p. 637; Lord Millet, ibid., p. 649; Lord Philipps of Worth Matravers, ibid., p. 661.

98. Opinion de Lord Philipps of Worth Matravers, Regina v. Bow Street Metropolitan Stipendiary Magistrate, ex parte Pinochet Ugarte ( ${ }^{\circ} 3$ ), House of Lords, 24 mars 1999, ILM, 1999, p. 660.

99. Opinion de Lord Browne-Wilkinson, Regina v. Bow Street Metropolitan Stipendiary Magistrate, ex parte Pinochet Ugarte ( $n^{\circ} 3$ ), House of Lords, 24 mars 1999, ILR, vol. 119, p. 156.

100. M. Cosnard, «La compétence universelle en matière pénale», p. 366-367.

101. Le Congo fait précisément valoir que la compétence universelle correspond à « un chef de compétence exceptionnel qui, dérogeant au principe de la souveraineté territoriale en matière pénale, ne tire sa conformité au droit international que du traité qui le prévoit. Par suite, il ne peut être opposé 


\section{La mise en œuvre concrète de la compétence universelle à l'égard de la torture}

Ces dernières années, la compétence universelle établie par la Convention contre la torture a connu une invocation croissante devant les juridictions nationales. Son application a cependant rencontré des obstacles, si l'on songe par exemple à l'affaire Hissène Habré au Sénégal qui n'a pas permis une mise en œuvre effective de la compétence universelle, principalement en raison des difficultés de transposition du droit international en droit interne. En l'espèce, suite au dépôt d'une plainte par des ressortissants tchadiens contre l'ex-président tchadien, Hissène Habré, résidant au Sénégal, auprès du doyen des juges d'instruction du tribunal régional de Dakar, le juge d'instruction du tribunal régional de Dakar a procédé, le 3 février 2000, à l'inculpation d'Hissène Habré pour " complicité de torture », sur le fondement de la Convention. Après plusieurs péripéties judiciaires, la Cour de cassation de Dakar a mis un terme définitif aux poursuites engagées à l'encontre d'Hissène Habré le 20 mars 2001, déclarant l'incompétence des juridictions sénégalaises. Pour la Cour, si le Sénégal a certes ratifié la Convention internationale contre la torture en 1986, il n'a cependant pas, conformément à son obligation conventionnelle, pris les mesures nécessaires pour établir le principe de la compétence universelle à l'égard de la torture dans son ordre juridique interne ${ }^{102}$.

La mise en œuvre de la compétence universelle a par contre rencontré plus de succès aux Pays-Bas ${ }^{103}$, au Royaume-Uni ${ }^{104}$ ou encore en France. Si l'on envisage plus précisément l'exemple français, l'exercice de la compétence universelle est doublement conditionné : il faut, d'une part, qu'un traité auquel la France est partie prévoie cette compétence, et, d'autre part, que les personnes suspectées d'avoir commis le crime en question se trouvent sur le territoire français. Ces deux conditions sont énoncées à l'article 689-1 du Code de procédure pénale. Les articles suivants mentionnent expressément les conventions internationales en application desquelles la compétence universelle peut être exercée par la France, l'article 689-2 du Code de procédure pénale prévoyant expressis verbis la compétence universelle des juridictions françaises à l'égard du crime de torture, cette disposition ayant été introduite à la suite de la ratification par la France de la Convention.

Après plusieurs affaires manifestant la réticence du juge français à effectuer une pleine et entière application de la compétence universelle conventionnelle en matière de torture ${ }^{105}$, la France semble s'être finalement engagée

qu'aux États parties à ce traité pour des faits commis sur le territoire de l'un d'eux, ainsi qu'à leurs ressortissants. Il est pour les autres États res inter alios acta. Or, le Congo n'est pas partie à la Convention de New York du 10 décembre 1984. Il est, dès lors, impossible d'opposer à cet État la règle de compétence écrite à l'article 689-2 du Code de procédure pénale français ». Voir Cour internationale de justice, Requête introductive d'instance enregistrée au Greffe de la Cour le 9 décembre 2002 et inscrite au rôle général de la Cour le 11 avril 2003, Certaines procédures pénales engagées en France (République démocratique du Congo c. France) : www.icj-cij.org/docket/files/129/7066.pdf, p. 8.

102. Selon la Cour de cassation, «aucun texte de procédure ne reconnaît une compétence universelle aux juridictions sénégalaises en vue de poursuivre et de juger, s'ils sont trouvés sur le territoire de la République, les présumés auteurs ou complices de faits qui entrent dans les prévisions de la loi du 28 août 1996 portant adaptation de la législation sénégalaise aux dispositions de l'article 4 de la Convention lorsque ces faits ont été commis hors du Sénégal par des étrangers ; [... la présence au Sénégal d'Hissène Habré ne saurait à elle seule justifier les poursuites intentées contre lui ». Affaire Hissène Habré, arrêt de la Cour de cassation de Dakar, arrêt n ${ }^{\circ}$ 14, 20 mars 2001. L'affaire a ensuite été relayée en Belgique où, après quatre ans d'investigations, un juge belge a émis un mandat d'arrêt international le 19 septembre 2005 visant l'ancien dictateur, conduisant à son arrestation au Sénégal le 15 novembre 2005. Le 25 novembre 2005, la cour d'appel de Dakar s'est cependant déclarée incompétente pour statuer sur la demande d'extradition déposée par la Belgique. Face à cette impasse juridique, le ministre des Affaires étrangères sénégalais, considérant qu'il appartenait à l'Union africaine d'indiquer la juridiction compétente pour connaître de cette affaire, a placé Hissène Habré à la disposition du président de l'Union africaine. C'est dans ce cadre que l'Union africaine a adopté, le 24 janvier 2006, une "décision sur le procès d'Hissène Habré et l'Union africaine ", mettant en place un groupe d'experts judiciaires chargé d'examiner les options disponibles afin de juger l'ancien dictateur. Sur la base des conclusions du groupe d'experts, soulignant que le Sénégal se trouvait dans l'obligation juridique de juger ou d'extrader l'ancien dictateur, l'Union africaine, lors de son

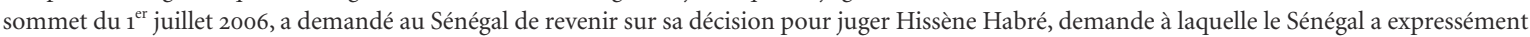
fait droit lors de ce même sommet. Deux ans après, le jugement de l'ancien président tchadien n'a cependant toujours pas débuté.

103. Les Pays-Bas ont ainsi mis en œuvre la compétence universelle en matière de torture suite au dépôt de plusieurs plaintes visant un ressortissant de la République démocratique du Congo, ancien commandant de la garde civile de Mobutu, soupçonné d'avoir commis des actes de torture sous le règne de Mobutu, et qui avait trouvé refuge aux Pays-Bas après la chute du régime. L'intéressé, Sebastien Nzapali, fut arrêté en septembre 2003 . Le 7 avril 2004, la Cour de district de Rotterdam l'a reconnu coupable de complicité d'actes de torture et l'a condamné à deux ans et demi d'emprisonnement. Public Prosecutor v. Sebastien Nzapali, Rotterdam District Court, 7 avril 2004. Sur cette affaire, voir M. T. Kamminga, « First Conviction under the Universal Jurisdiction Provisions of the UN Convention against Torture : Rotterdam District Court, 7 April 2004, Sebastien N. », Netherlands International Law Review, n ${ }^{\circ}$, 2004, p. 439-449.

104. On peut notamment citer le jugement, par un tribunal londonien, d'un ancien chef de guerre afghan, Faryadi Sarwar Zardad, qui était arrivé sur le territoire britannique en 1998 avec l'intention de demander l'asile politique. Interpellé en 2002, il a été jugé en application de la compétence universelle prévue par la Convention contre la torture, à laquelle tant le Royaume-Uni que l'Afghanistan sont parties. Le 18 juillet 2005 , il a été condamné à vingt ans de réclusion pour actes de torture commis pendant la guerre civile dans son pays à l'encontre de ressortissants afghans.

105. Il faut sur ce point brièvement mentionner l'affaire dite du prêtre rwandais, concernant des poursuites intentées en France par des ressortissants rwandais à l'encontre d'un prêtre rwandais, Wenceslas Munyeshyaka, qui se trouvait en France et auquel il était reproché d'avoir commis des actes de génocide et de torture lors du génocide rwandais de 1994. Dans un premier temps, le juge d'instruction du Tribunal de grande instance de Privas rendit une ordonnance d'incompétence partielle, se déclarant incompétent sur la base de la Convention sur le génocide en ce que celle-ci ne consacre pas la compétence universelle et fonda justement sa compétence sur la Convention contre la torture. Suite à l'appel de cette décision, la cour d'appel de Nîmes infirma cependant cette ordonnance dans un arrêt du 20 mars 1996. Relevant que le prêtre rwandais était poursuivi d'une part pour "génocide» et, d'autre part, pour « torture», elle considéra en effet «que la compétence du juge d'instruction doit s'apprécier au regard de la plus haute acception pénale, la plus spécifique, celle de génocide, infraction distincte des tortures et actes de barbarie prévus par la Convention des Nations unies du 10 décembre 1984 [...], que la Convention du 9 décembre 1948 sur le génocide ne prévoit aucune règle de compétence universelle, qu’elle donne compétence aux tribunaux de l'État sur le territoire duquel l'acte a été commis ou à une Cour criminelle internationale » (Wenceslas Munyeshyaka 
dans la voie du respect de ses obligations conventionnelles. Le premier procès achevé en application de la compétence universelle à l'égard d'actes de torture date de 1999 et s'inscrit dans le cadre de la dynamique créée par l'affaire Pinochet. Il s'agit de l'affaire Ely Ould Dah ${ }^{106}$, relative à l'application de la Convention de 1984 contre la torture. Le 4 juin 1999, la Fédération internationale des Ligues des Droits de l'homme a engagé une procédure auprès du procureur de la République de Montpellier, sollicitant l'ouverture d'une information judiciaire à l'encontre d'un officier mauritanien, pour des actes de torture qu'il aurait commis entre 1990 et 1991 sur le territoire mauritanien, à l'encontre de Mauritaniens, réfugiés politiques en France. Le capitaine des forces armées mauritaniennes, Ely Ould Dah, effectuait à l'époque un stage de perfectionnement dans une école militaire de Montpellier. Après avoir été mis en examen par un tribunal de Montpellier pour « crimes de torture » et placé en détention en juillet 1999, la chambre d'accusation de la cour d'appel de Montpellier décida cependant de remettre l'officier mauritanien en liberté, en le plaçant sous contrôle judiciaire. Bien que le capitaine mauritanien ait fui la France pour rejoindre la Mauritanie en avril 2000, le juge d'instruction près du Tribunal de grande instance de Montpellier rendit, le 28 mai 2001, une ordonnance de mise en accusation, faisant valoir qu'il appartenait à la France, «comme État signataire de la Convention de New York, de se saisir des faits [...] susceptibles d'entrer dans le champ d'application de cette Convention ». Le 8 juillet 2002, la chambre de l'instruction de la cour d'appel de Nîmes ordonna ainsi la mise en accusation du capitaine mauritanien devant la cour d'assises du Gard pour les actes de torture et barbaries commis en Mauritanie ${ }^{107}$. Suite au rejet du pourvoi en cassation de l'officier mauritanien par la Cour de cassation le 23 octobre $2002^{108}$, renvoyant l'affaire devant la cour d'assises du Gard, le procès de l'intéressé s'est finalement tenu en France en 2005. Le $1^{\text {er }}$ juillet 2005, la cour d'assises de Nîmes l'a reconnu coupable d'avoir «commis directement, ordonné et organisé des actes de torture» et l'a condamné à dix ans de réclusion. Même si ce procès s'est déroulé in absentia, il fait figure de précédent face aux atermoiements passés des juridictions françaises dans des affaires concernant des ressortissants rwandais et yougoslaves ${ }^{109}$. Dans le même ordre d'idées, le 15 décembre 2008, sur le fondement de la compétence universelle établie dans la Convention internationale contre la torture, la cour d'assises du Bas-Rhin a jugé et condamné à huit ans d'emprisonnement l'ancien vice-consul de Tunisie à Strasbourg, Khaled Ben Saïd, le reconnaissant coupable d'avoir commis des actes de torture à l'encontre d'une ressortissante tunisienne en 1997, alors qu'il était commissaire de police à Jendouba ${ }^{110}$. Le procès s'est déroulé selon la procédure de défaut criminel, Khaled Ben Saïd ayant fui vers la Tunisie en 2001 après avoir appris qu'une plainte avait été déposée à son encontre. Il faut aussi mentionner l'affaire précitée des Disparus $d u$ Beach $^{111}$. Après plusieurs péripéties judiciaires, notamment l'annulation, le 22 novembre 2004, de l'ensemble de l'instruction conduite à Meaux par la chambre de l'instruction de la cour d'appel de Paris, la Cour de cassation a ordonné, le 10 janvier 2007 , la reprise de la procédure, notamment sur le fondement de la Convention contre la torture ${ }^{112}$.

Si la compétence universelle et sa mise en œuvre effective constituent une perspective particulièrement intéressante en matière de lutte contre l'impunité des tortionnaires, il convient cependant de rester conscient du fait que son application effective ${ }^{113}$ est, dans la pratique,

(affaire du prêtre rwandais), Arrêt de la cour d'appel de Nîmes, Chambre d'accusation, 20 mars 1996, RGDIP, p. 1084-1089). Le raisonnement opéré par la cour d'appel est critiquable en ce qu'il revient à considérer que si des actes identiques sont susceptibles de tomber sous le coup à la fois de la qualification de "génocide» et de celle de «torture», le juge français ne peut, contre toute logique, être compétent que pour connaître des faits de génocide. Il s'agit là d'une manière à peine voilée de refuser la mise en œuvre de la compétence universelle. Dans la mesure où le crime de génocide ne fait pas l'objet d'une attribution de compétence universelle au profit des juridictions françaises, celles-ci sont en effet conséquemment incompétentes pour statuer sur l'affaire. Sur pourvoi formé par le procureur général, la chambre criminelle de la Cour de cassation a, en bonne logique, censuré le raisonnement opéré par la cour d'appel et cassé son arrêt. Le 6 janvier 1998, elle a en effet relevé qu' «il suffit que les faits puissent être appréhendés sous une qualification conférant compétence aux juridictions françaises pour que celles-ci puissent instruire, même si les faits peuvent également être appréhendés sous une qualification plus grave ou plus spécifique, que la juridiction française est compétente pour connaître d'une infraction commise à l'étranger par un étranger, dès lors qu'elle recouvre les mêmes faits que ceux qui, sous une autre qualification, ressortissent de sa compétence" (Arrêt de la Cour de cassation, chambre criminelle, 6 janvier 1998, RGDIP, n 3, 1998, p. 825-828). Ce faisant, la Cour de cassation a ordonné la reprise des poursuites contre le prêtre rwandais pour torture et pour génocide, suite à l'entrée en vigueur en France de la loi portant adaptation de la législation française avec le Statut du Tribunal pénal international pour le Rwanda, établissant la compétence universelle des juridictions françaises à l'égard du crime de génocide.

106. Sur cette affaire, voir FIDH, Mauritanie: affaire Ely Ould Dah. Développements et enjeux relatifs à l'affaire Ely Ould Dah, rapport établi par le groupe d'action judiciaire de la FIDH : www.fidh.org/IMG/pdf/ely2005f.pdf.

107. La Chambre affirmant que «l'article 689-2 du Code de procédure pénale [...] donne compétence à la juridiction française pour poursuivre ou juger, s'il est trouvé en France, quiconque, qui, hors du territoire de la République, s'est rendu coupable de faits qualifiés crime ou délit qui constituent des tortures et autres peines ou traitements cruels ou dégradants, au sens de l'article $1^{\text {er }}$ de la Convention de New York du 10 décembre 1984, en vigueur en France depuis le 26 juin 1987 ».

108. Affaire Ely Ould Dah, Cour de cassation, chambre criminelle, 23 octobre 2002, RGDIP, n 2, 2003, p. 309-312.

109. Voir B. Stern, «La compétence universelle en France : le cas des crimes commis en ex-Yougoslavie et au Rwanda », German Yearbook of International Law, vol. 40, 1997, p. 280-299.

110. Voir F. Beaugé, "Huit ans de prison pour le diplomate tunisien reconnu coupable de tortures », Le Monde, 17 décembre 2008.

111. Sur cette affaire, voir A. Giudicelli, «Compétence universelle : caractère suffisant de la présence en France de l'une des personnes soupçonnées d'avoir commis les faits (Crim., 10 janvier 2007, $\mathrm{n}^{\circ}$ 04-87-245, Bull. crim., $\mathrm{n}^{\circ} \mathrm{7}$ ) », Revue de science criminelle et de droit comparé, $\mathrm{n}^{\circ} 3,2007, \mathrm{p} .566-572$.

112. Cour de cassation, Chambre criminelle, arrêt du 10 janvier 2007.

113. De manière générale, voir M. Delmas-Marty, «La responsabilité pénale en échec (prescription, amnistie, immunités) », in Juridictions nationales et crimes internationaux, A. Cassese et M. Delmas-Marty (dir.), Paris, PUF, 2002, p. 613-652. 
bien souvent neutralisée par de nombreux obstacles juridiques. La difficile transposition de la compétence universelle dans les ordres juridiques internes, la réticence du juge interne à se comporter en véritable agent de la communauté internationale, les obstacles procéduraux relatifs à l'opposabilité de la prescription ${ }^{114}$, de l'amnistie et surtout de l'immunité de juridiction reconnue aux dirigeants étatiques ${ }^{115}$, s'opposent souvent à une pleine et entière application de la compétence universelle. À cela s'ajoutent encore des obstacles politiques qui ont trait à la préoccupation des États de ne pas entraver les relations diplomatiques avec certains États étrangers ${ }^{116}$.

\section{B. Le droit de la victime à la réparation du préjudice subi}

La Convention contre la torture prend dûment en considération le fait que la réparation de ces actes et leur indemnisation peuvent favoriser la réhabilitation des victimes. L'article 14 de la Convention contre la torture est particulièrement explicite sur ce point. Il dispose en effet que: «1. [t] out État partie garantit, dans son système juridique, à la victime d'un acte de torture, le droit d'obtenir réparation et d'être indemnisée équitablement et de manière équitable, y compris les moyens nécessaires à sa réadaptation la plus complète possible. En cas de mort de la victime résultant d'un acte de torture, les ayants cause de celle-ci ont droit à indemnisation. 2. Le présent article n'exclut aucun droit à indemnisation qu'aurait la victime ou toute autre personne en vertu des lois nationales. »

C'est dans le cadre de l'affirmation générale d'un « droit à réparation » au profit des victimes de graves violations des Droits de l'homme que certains auteurs ont interprété cette disposition comme une reconnaissance de la compétence universelle en matière civile ${ }^{117}$, permettant à des victimes étrangères d'actes de torture, commis à l'étranger par des étrangers, d'intenter une action en réparation devant les tribunaux d'États tiers.

Dans l'affaire Furundzija, le TPIY a, par exemple, fait une brève référence à la notion de compétence civile universelle. Après avoir conclu que l'interdiction de la torture revêtait le caractère de jus cogens, il a déterminé les différents effets juridiques qui s'attachaient à cette qualification. Il a notamment fait valoir qu'en cas d'adoption par un État de mesures nationales qui autoriseraient ou toléreraient la pratique de la torture, «les victimes potentielles pourraient, si elles en ont la capacité juridique, [...] engager une action en réparation auprès d'une juridiction étrangère qui serait invitée de la sorte [...] à ne tenir aucun compte de la valeur juridique de l'acte national autorisant la torture ${ }^{118}$. Si l'on peut considérer que l'article 14 n'interdit pas aux États d'établir dans leur droit interne un droit de recours devant leurs juridictions civiles au bénéfice d'étrangers victimes, à l'étranger, d'actes de torture, il paraît cependant difficile d'aller jusqu'à affirmer qu'il prévoit l'obligation à la charge des États parties d'établir dans leur ordre juridique interne un chef de compétence civile universelle, sauf à procéder à une interprétation sollicitée des dispositions de la Convention. L'article 14 est en effet généralement interprété comme établissant à la charge de l'État partie l'obligation de prévoir dans son ordre juridique interne un droit d'action civile pour les victimes d'actes de torture qui ont été commis dans un territoire relevant de sa juridiction et non à raison d'actes de torture qui ont été commis à l'étranger.

On comprend aisément l'intérêt que suscite cette déclinaison de la compétence universelle en matière civile, placée au service d'une justice réparatrice pour les victimes de violations des Droits de l'homme. Aux ÉtatsUnis, la compétence universelle trouve d'ailleurs un écho en matière civile: sur le fondement de l'Alien Tort Claims Act ${ }^{119}$, des actions en réparation peuvent en effet être

114. Il faut, sur ce point, relever la divergence d'appréciation existant entre les juges internationaux et certaines autorités nationales. S'agissant du crime de torture, le TPIY a estimé, dans l’affaire Furundzija précitée, que «la torture est sans doute imprescriptible " $\$ 157$ du jugement), cette considération découlant selon les juges de la nature impérative de l'interdiction de la torture. Pour autant, si le crime de torture est considéré comme imprescriptible dans certains États, il est, dans la plupart des États, soumis aux règles du droit commun de la prescription.

115. Pour ne prendre que l'exemple de la France, on peut citer le traitement de la plainte déposée le 25 octobre 2007 par plusieurs associations de défense des Droits de l'homme contre Donald Rumsfeld, ancien secrétaire d'État américain à la défense. Cette plainte visait Donald Rumsfeld du chef d'actes de torture pour avoir directement et personnellement ordonné le recours à des méthodes poussées d'interrogatoires constitutives de tortures, mises en œuvre sous sa supervision, notamment dans les centres de détention de Guantánamo et d'Abu Ghraïb entre octobre 2002 et avril 2003 , en violation de la Convention internationale contre la torture. La plainte a fait l'objet d'un classement sans suite par le procureur de la République près le Tribunal de grande instance de Paris au motif que Donald Rumsfeld bénéficiait, même après la cessation de ses fonctions, d'une immunité de juridiction, en application du droit international coutumier tel que reconnu par la Cour internationale de justice, pour les actes accomplis à titre officiel dans l'exercice de ses fonctions. Voir L. Balmond, «France / États-Unis. Plainte pour torture contre Donald Rumsfeld, 25 octobre 2007 », RGDIP, $\mathrm{n}^{\circ} 1,2008$, p. 153-155.

116. Ainsi que le relève un auteur, "[s]ometimes the obstacle is political interference [...]. Political officials are accused of preventing the exercise of universal jurisdiction by other means, such as by tipping off suspects about investigations or even assisting them to leave the country» (C. Hall, " Universal Jurisdiction : New Uses for an Old Tool », in Justice for Crimes against Humanity, M. Lattimer et P. Sands (éd.), Oxford, Hart Publishing, 2003, p. 6263). L'attitude des autorités françaises lors de la visite du général algérien Nezzar à Paris illustre ce point de vue. Le 25 avril 2001 , suite au dépôt d'une plainte à son encontre pour torture, traitements cruels, inhumains et dégradants devant le procureur de la République de Paris, le Parquet de Paris avait décidé d'ouvrir une enquête. Dans la nuit du 25 au 26 avril 2001, le général Nezzar a cependant précipitamment quitté le territoire français sans que la police française ne puisse l'interroger. Le 14 juin 2001, constatant que le général Nezzar n'était plus sur le territoire français, le Parquet a classé la plainte sans suite.

117. D. F. Donovan et A. Roberts, «The Emerging Recognition of Universal Civil Jurisdiction », American Journal of International Law, ${ }^{\circ}$ 1, $2006, \mathrm{p} .148$. 118. TPIY, Le Procureur c. Anto Furundzija, $\$ 155$. Nous soulignons.

119. Alien Tort Claims Act (ATCA), 28 USC, $\$ 1350$. Sur cette loi, voir I. Moulier, «Observations sur l'Alien Tort Claims Act et ses implications internationales ", $A F D I, 2003$, p. 129-164. 
intentées devant les tribunaux fédéraux, par des victimes de nationalité étrangère (non américaines), pour des violations du droit international commises à leur encontre, sur le territoire d'États tiers, par des étrangers. Conçus comme un pis-aller ou une alternative à des procès pénaux, ces procès civils ne peuvent donner lieu à des sanctions pénales; ils visent seulement à la réparation d'un préjudice exprimée en termes financiers, sous forme de l'octroi de dommages et intérêts. La décision rendue en l'affaire Filartiga $v$. Peña-Irala ${ }^{120}$, jugée en 1980 par les juridictions américaines, a, à cet égard, constitué le point de départ des actions civiles en dommages et intérêts initiées devant les tribunaux américains par des étrangers contre des étrangers pour des faits commis à l'étranger. En l'espèce, les juridictions américaines se prononcèrent en faveur de la recevabilité d'une action civile en dommages et intérêts intentée par deux citoyens paraguayens, membres de la famille d'un jeune ressortissant paraguayen qui avait été torturé, au Paraguay, par un fonctionnaire de police paraguayen. Les juridictions américaines firent valoir que la pratique de la torture par un fonctionnaire étranger à l'encontre de l'un de ses concitoyens violait le droit international coutumier et pouvait par là même donner lieu à une action en réparation devant les tribunaux fédéraux américains sur le fondement de l'Alien Tort Claims Act ${ }^{121}$. Le tribunal fédéral de première instance (district est de New York) condamna par la suite l'officier de police paraguayen à verser près de 10,4 millions de dollars de dommages et intérêts aux demandeurs ${ }^{122}$.

Cette jurisprudence a par la suite été codifiée par le Torture Victim Protection Act ${ }^{123}$. Cette législation, adoptée par le Congrès américain le 12 juin 1992, permet aux ressortissants étrangers d'intenter une action civile en dommages et intérêts devant les juridictions américaines, à l'encontre des auteurs d'actes de torture ou d'exécutions extrajudiciaires, quelle que soit leur nationalité et quel que soit le lieu où les actes ont été commis ${ }^{124}$.

Pour autant, cette forme hybride de compétence universelle n'est pas, à ce jour, ancrée dans le droit international positif. Elle demeure en effet une spécificité américaine et s'avère sans précédent, aucun autre droit national ne s'étant, à ce jour, orienté vers la reconnaissance, au profit des juridictions nationales, d'une compétence civile universelle. Si certains auteurs n'ont pas manqué d'appeler à une «européanisation de la compétence civile universelle ${ }^{125}$ telle qu'elle est pratiquée par les juridictions américaines, l'arrêt rendu en 2001 par la Cour européenne des Droits de l'homme dans l'affaire $A l-A d s a n i^{126}$ n'ouvre guère la voie à cette perspective ${ }^{127}$. La Cour a en effet considéré qu'un État partie ne saurait être tenu, aux termes des dispositions de la Convention, d'offrir à ses ressortissants une voie de recours judiciaire en matière civile pour des actes de torture commis par les autorités d'un État étranger à leur encontre en dehors de sa juridiction ${ }^{128}$, soit en dehors de son territoire. La Cour a ainsi écarté toute obligation pour les États parties d'établir dans leur arsenal juridique interne un chef de compétence civile universelle aux fins de réparation de dommages liés à des actes de torture commis en territoire étranger, l'absence d'une telle voie de recours n'étant pas considérée contraire à la Convention tant au regard du droit à un recours effectif qu'au regard du droit d'accès à un tribunal.

Ce panorama général du cadre juridique de la lutte contre la torture met en évidence l'importance essentielle que l'interdiction de la torture, énoncée à l'article 5 de la Déclaration universelle des Droits de l'homme, a depuis acquise sur un plan normatif. L'interdiction a en effet progressivement été consolidée pour être désormais

120. Dolly M. E. Filartiga and Joel Filartiga v. Americo Norberto Peña-Irala, 630 F.2d 876 ( ${ }^{\text {d }}$ Cir. 1980), ILR, vol. 77, p. 169-184. Voir J. M. Blum et R. G. Steinhardt, "Federal Jurisdiction over International Human Rights Claims: The Alien Tort Claims Act after Filartiga v. Peña-Irala ", Harvard International Law Journal, n 1, 1981, p. 53-113 ; J. P. George, «Defining Filartiga: Characterizing International Torture Claims in United States Courts », Dickinson Journal of International Law, $\mathrm{n}^{\circ} 1,1984$, p. 1-42.

121. "Construing this rarely-invoked provision [ 28 USC \$1350], we hold that deliberate torture perpetrated under color of official authority violates universally accepted norms of the international law of human rights, regardless of the nationality of the parties. Thus, whenever an alleged torturer is found and served with process by an alien within our borders, $\$ 1350$ provides federal jurisdiction. » Filartiga v. Peña-Irala, p. 878.

122. Filartiga v. Peña-Irala, 577 F. Supp. 860 (EDNY 1984), ILR, vol. 77, p. 185-191. Satisfaction symbolique qui n'eut cependant aucune conséquence pour les demandeurs, le défendeur ne disposant pas de fonds aux États-Unis.

123. Torture Victim Protection Act (TVPA), 1991, Pub. L., n 102-256, 106 Stat. 73 (1992), 28 USC $\$ 1350$. Sur cette législation, voir H. R. D. Cudahy, "Symbolic Justice: Alien Torture Victims in United States Courts », RIDP, n 1-2, 1996, p. 299-317.

124. On relèvera que le Torture Victim Protection Act n'est pas la législation de transposition de la Convention des Nations unies contre la torture. Les États-Unis ont signé la Convention le 18 octobre 1988 mais ne l'ont cependant ratifiée que le 21 octobre 1994, soit postérieurement à l'adoption du TVPA. De plus, les États-Unis ont donné une interprétation stricte de l'article 14 de la Convention contre la torture lors de la ratification de la Convention. Ils ont en effet précisé, dans une déclaration interprétative, que «l'article 14 fait obligation à tout État partie de garantir à toute personne le droit d'obtenir réparation pour le préjudice qui lui a été causé dans le seul cas où les actes de torture ont été commis dans le territoire sous la juridiction de cet État partie ", soit à raison d'actes de torture commis sur son territoire et non à raison d'actes de torture commis à l'étranger. Voir Nations unies, Comité contre la torture, Examen des rapports présentés par les États parties conformément à l'article 19 de la Convention. Rapports initiaux que les États parties devaient présenter en 1995, additif, États-Unis d’Amérique (rapport du 15 octobre 1999), CAT/C/28/Add.5, 9 février $2000, \$ 268$, p. 66.

125. J.-F. Flauss, «La compétence civile universelle devant la Cour européenne des Droits de l'homme. Note sous CEDH, Al Adsani c. Royaume-Uni, Grande Chambre, 21 novembre 2001 ", RTDH, n 53, 2003, p. 156-175(163).

126. CEDH, Al Adsani c. Royaume-Uni, req. n³ 35763/97, arrêt du 21 novembre 2001.

127. Voir la note critique d'O. de Frouville, «Droit à un tribunal et immunités de juridiction. Note sous CEDH, Al-Adsani c. Royaume-Uni, Fogarty c. Royaume-Uni, McElhinney c. Irlande, 21 novembre 2001 ", JDI, n 1, 2002, p. 273-279.

128. «Le requérant ne prétend pas que les actes de torture qu'il aurait subis aient été perpétrés dans la juridiction du Royaume-Uni ou que les autorités britanniques aient un lien de causalité avec eux. Dans ces conditions, on ne saurait dire que la Haute Partie contractante était tenue de lui offrir une voie de recours civile pour les tortures que les autorités koweïtiennes lui auraient infligées.» (Ibid., \$40). 
considérée, avec l'interdiction du génocide, comme l'une des rares normes de jus cogens internationalement reconnues. L'article 5 de la Déclaration universelle a incontestablement constitué le socle de l'édifice juridique de la lutte internationale contre la torture qui n'a depuis cessé de se développer. La criminalisation de la torture, la mise en place de mécanismes sophistiqués et renforcés de prévention de la torture et la consécration de la compétence universelle constituent des moyens propres à donner véritablement corps à l'interdiction de principe énoncée à l'article 5 de la Déclaration.

Bien que l'interdiction des actes de torture soit universelle et absolue, la torture et les autres traitements cruels, inhumains ou dégradants n'en demeurent pas moins monnaie courante dans de nombreux États. Loin de disparaître, la torture connaît en effet des formes renouvelées, voire une forme de «banalisation » dans le contexte de la lutte contre le terrorisme au nom des exigences sécuritaires. Si l'on ne peut ainsi que déplorer la dichotomie et le paradoxe qui existent entre l'interdiction absolue de la torture et la persistance de sa pratique, on ne peut toutefois nier les avancées du droit positif en matière de lutte contre la torture. Au regard du cadre juridique de la lutte contre la torture qui s'avère particulièrement complet et abouti, il faut conclure que la persistance de la torture est loin de découler d'un vide juridique. Elle résulte sans conteste d'un manque de volonté politique des États de s'acquitter des obligations qui leur incombent en vertu du droit international et particulièrement en vertu de la Convention internationale contre la torture.

Dans le contexte actuel de la lutte internationale contre le terrorisme, le rappel inlassable du caractère fondamental et indérogeable de l'interdiction de la torture s'avère plus que jamais nécessaire, sauf à ce que l'article 5 de la Déclaration universelle des Droits de l'homme et tous les efforts subséquemment menés pour lutter contre la torture ne soient totalement vidés de leur substance. Il convient dans ce cadre de garder présents à l'esprit les propos du juge américain, Sandra Day O'Connor, formulés lors de l'affaire José Padilla, jugée par la Cour suprême des États-Unis, le 28 juin 2004: « [1]'enjeu [...] n'est rien moins que celui de l'essence d'une société libre. Si cette nation reste attachée aux idéaux symbolisés par son drapeau, elle ne doit pas utiliser les armes des tyrans pour résister à un assaut des forces de la tyrannie. » Ce rappel constitue en effet un premier jalon, aussi modeste soit-il, vers une prise de conscience accrue des exigences du droit international contemporain, qui constitue un préalable indispensable à sa mise en œuvre effective. » 